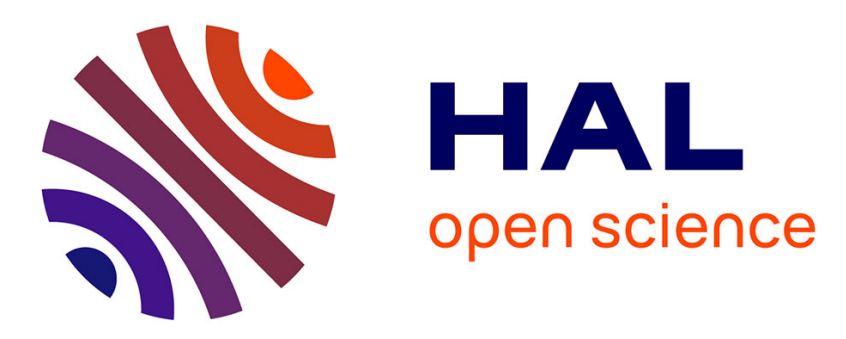

\title{
Validity limits of the passive treatment of impurities in gyrokinetic tokamak simulations
}

\author{
Maxime Lesur, Chabha Djerroud, Kyungtak Lim, Etienne Gravier, Malik \\ Idouakass, Jérôme Moritz, Julien Médina, Thierry Réveillé, Thomas Drouot, \\ Thomas Cartier, et al.
}

\section{To cite this version:}

Maxime Lesur, Chabha Djerroud, Kyungtak Lim, Etienne Gravier, Malik Idouakass, et al.. Validity limits of the passive treatment of impurities in gyrokinetic tokamak simulations. Nuclear Fusion, 2020, 60 (3), pp.036016. 10.1088/1741-4326/ab6e48 . hal-02485083

\section{HAL Id: hal-02485083 https://hal.science/hal-02485083}

Submitted on 24 Feb 2020

HAL is a multi-disciplinary open access archive for the deposit and dissemination of scientific research documents, whether they are published or not. The documents may come from teaching and research institutions in France or abroad, or from public or private research centers.
L'archive ouverte pluridisciplinaire HAL, est destinée au dépôt et à la diffusion de documents scientifiques de niveau recherche, publiés ou non, émanant des établissements d'enseignement et de recherche français ou étrangers, des laboratoires publics ou privés. 


\title{
Validity limits of the passive treatment of impurities in gyrokinetic Tokamak simulations
}

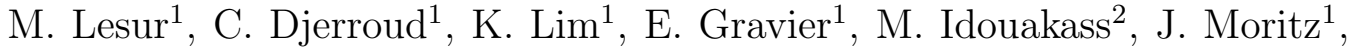 \\ J. Médina ${ }^{1}$, T. Réveillé ${ }^{1}$, T. Drouot ${ }^{1}$, T. Cartier-Michaud ${ }^{3}$, X. Garbet $^{4}$ \\ ${ }^{1}$ Institut Jean Lamour, Université de Lorraine,

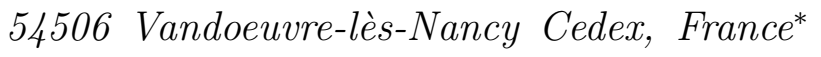 \\ ${ }^{2}$ NIFS, Tajimi, Japan \\ ${ }^{3}$ CNRS, M2P2, 13451 Marseille, France and \\ ${ }^{4}$ CEA, IRFM, 13108 Saint-Paul-Lèz-Durance Cedex, France
}

(Dated: January 13, 2020)

\begin{abstract}
In gyrokinetic simulations of turbulent impurity transport, trace impurity species are often treated as passive species, in the sense that they are not included in Maxwell equations. This is consistent with the assumption that impurities with low enough concentrations are impacted by turbulence generated by electrons and main ions, but do not impact it significantly in return. In this work, we relax this assumption, and investigate the active impacts of impurity on impurity transport as a function of its concentration, in the presence of trapped-particle-driven turbulence. We focus on $W^{40+}$ tungsten, which is relevant for modern tokamaks, and adopt a reduced gyrokinetic bounce-averaged model for trapped particles in a simplified tokamak geometry. The impacts depend on the relationship between equilibrium density gradient and temperature gradient. When these gradients are equal, we observe that tungsten can be treated as a passive species for concentrations below $2 \times 10^{-4}$. Above this concentration, the impurity significantly impacts both density and heat transport, essentially quenching them for concentrations above $10^{-3}$. This quenching occurs as electric potential fluctuations become in phase with impurity density fluctuations.
\end{abstract}

\footnotetext{
* maxime.lesur@univ-lorraine.fr
} 


\section{INTRODUCTION}

The ideal plasma for fusion reactions in the core of ITER and future reactors is a mixture of deuterium and tritium. However, core Tokamak plasmas are often contaminated by other ion species, called impurities. Except for Helium (fusion products), impurities originate from the edge. Nitrogen, neon and/or argon particles are injected to mitigate hot spots on walls. The plasma can erode plasma-facing components, such as the walls of the confinement chamber and the divertor, which can be made of beryllium, carbon, or tungsten. For typical plasma parameters, these impurities are transported from the edge to the core by both collisional (neoclassical) and turbulent processes. Accumulation of impurities in the core threatens the viability of fusion. One issue is the dilution of fuel, which degrades the efficiency of fusion reactors. Another urgent issue is that heavy impurities, which are not fully ionized, radiate away the energy generated by fusion.

The efficiency of fusion is very sensitive to core impurity concentration. For example, a reduction by a factor 4 of tungsten concentration in the core, from $12 \times 10^{-5}$ to $3 \times 10^{-5}$ would lead to a $40 \%$ decrease in the minimum value of triple product required for ignition, and a $40 \%$ decrease in the required temperature [1]. Since slight contamination in the core can yield prohibitive energy losses, it is crucial to improve our lacking understanding of heavy impurity transport.

In particular, ITER will feature a tungsten divertor. This choice of material was ratified relatively recently (2013-14), based on its behavior under extreme heat fluxes. In response, global efforts are focusing on the prediction of core tungsten density. Tokamak experiments such as JET and ASDEX-Upgrade indicate significant tungsten accumulation in the core [2, 3]. Promising remedies have been discovered empirically (See [4] for a partial review). For example, the peaking of tungsten density profile can be reduced by increasing central radiofrequency heating power in ASDEX Upgrade [5]. Control of Edge-Localized Modes is another important mitigation scheme [6] 8]. Impurity transport from edge to core appears to depend

on key plasma parameters such as the collisionality (a normalized collision frequency), the rate of toroidal rotation of the plasma, the concentration of impurities, and the mass and charge of each impurity species. First-principle numerical simulations are often used to explore this vast domain.

Quantitatively-accurate transport modelling of core tokamak plasmas requires a 5D gy- 
rokinetic description [9]. Developments of gyrokinetic simulation codes have been the focus of many research teams worldwide [10]. The approach is now mature enough to provide critical understandings and predictions of experiments [11-13].

For electrons and deuterium ions, drift-wave turbulence is the dominant source of crossfield transport of particles and energy in tokamaks, dwarfing neoclassical collisional transport. However, the transport of high- $Z$ impurities poses a specific challenge. The neoclassical and the turbulent contributions can be comparable. Most codes treat each channel separately, with a neoclassical code for collisions, and a gyrokinetic code for turbulence. A recent, growing body of evidence shows that collisional and turbulent channels couple synergistically [14]. When including both processes self-consistently, impurity transport is found to be significantly different from a simple sum of the two contributions (discrepancies can reach and overcome $100 \%$, yielding either over-prediction or under-prediction depending on the region of the plasma). More recently, impurity transport was investigated by global gyrokinetic simulations with an accurate collision operator, which fully recovers neoclassical theory [15]. Due to the new collision operator, and to the difference of scales between deuterium and tungsten, the cost of one simulation is of the order of 2 to 3 million core.hours on modern supercomputers [16].

The concentration of impurities in tokamaks is often small enough that the impurity contribution in the quasineutrality equation is often considered negligible. Indeed, the relative contribution of impurity density in quasineutrality is of the order of $C Z^{2}$, where $C$ is the impurity concentration, and $Z$ its charge number. This is the most used simplification in the published literature of turbulent transport. In the "trace" limit, $C Z^{2} \ll 1$, the impurity behaves as a passive species, or test-particle, which means that its presence does not affect the turbulent state. Several numerical simulations have been performed with passive impurity, showing for instance that the background turbulence can transport the impurity by diffusion and particle pinch velocities [17]. In this case the diffusion coefficient and the pinch velocity do not depend on the density gradient of the trace, but depend on the parameters of the background plasma. Therefore a diffusion coefficient and a pinch velocity can be determined unambiguously because of the linear relationship between flux and gradient. The passive treatment of impurities was also used for instance to investigate impurity advection in tokamak edge plasmas based on the two-dimensional Hasegawa-Wakatani model [18]. As another example, using the gyrokinetic GENE code in the case of nonlinear simulations of 
TEM turbulence [19] driven by steep density gradients and with passive impurities, it has been shown that the impurity peaking factor is weakly dependent on impurity charge. This code has also been used to calculate impurity transport coefficients due to ITG and TEM turbulence in JET, again considering passive impurities as the impurity fraction is very low $\left(10^{-6}\right)$ [20]. In this article the results are found to be in qualitative agreement with the experimental findings for the impurities Ne, Ar and Ni. For carbon, however, a discrepency between numerical results and experiment are observed, and these results may indicate that some important ingredient is missing in the models. Finally, using the GKW code in the trace limit [21], turbulent convection of heavy impurities in tokamak plasmas has been investigated, motivated by the expected increasing importance of the turbulent transport of heavy impurities for the ITER baseline scenario and a reference reactor (DEMO) plasma. The same code in the trace limit is used to study W impurity transport in JET and ASDEX upgrade [3]. Therefore, the passive treatment of impurities in the trace limit is widely adopted.

However, impurities can have a non-negligible active influence on turbulence [22]. In Ref. [23], impurity transport obtained from a self-consistent treatment is compared with the results of the trace impurity approximation for ITER-like profiles using a quasilinear fluid model. This indicates that a self-consistent treatment may be needed for $C Z^{2}>0.7$, particularly in the core which is dominated by TEM turbulence. Another, global fluid model was used to show that nickel impurities at concentrations of the order of $10^{-3}\left(C Z^{2}=23.5\right)$ can qualitatively alter transport properties [24]. In numerical studies based on gyrokinetics, non-trace impurities isotopic effects have been observed on the quasi-linear turbulent flux, although isotopic dependence of the instability linear growth rate is weak [25]. Moreover, it has been shown that the effects of impurity concentration on the linear growth rate can depend on impurity density gradient, using a gyrokinetic integral eigenmode equation in toroidal geometry [26, 27], or performing nonlinear bounce-average gyrokinetic simulations [28]. Recent numerical simulations performed with the GENE code [29] have shown that the light impurities cannot be considered as traces, as they have a non-negligible influence on the linear growth rate of the instability. Main ions and electrons as well as the impurity species are retained as active species. The W-concentration in JET ITER-like Wall experiments can reach $10^{-4}$ in the L-mode [30]. In order to study JET H-mode plasmas, in which the W concentration is also found to be of the order of $10^{-4}$, the GKW code has been used in the 
trace limit [2]. But central concentrations up to $10^{-3}$ are reached during the accumulation phase. For this reason, simulations in which $\mathrm{W}$ is not treated as passive have been performed in order to also investigate the difference with the trace limit. Calculations have shown that the passive and active limits differ only where the strong accumulation takes place.

In this paper, we aim mainly at estimating the range of validity of the passive treatment of high- $Z$ impurities. We focus on the turbulent transport of $W^{40+}$ tungsten. We run a series of simulations with self-consistent (active) treatment of impurities for a wide range of concentrations, and compare the results with a series (for better statistics) of simulations with passive impurities.

In conventional gyrokinetics, the dynamics is described in a 4D phase-space parameterized by one adiabatic invariant. If we limit the kinetic description to magnetically trapped particles, and rely on assumptions described in Section II], the bounce-averaged dynamics can be described in a reduced 2D phase-space, parameterized by the energy. The bounceaveraged gyrokinetic model for trapped particle precession resonance-driven turbulence [3135 is much more numerically tractable, and allows parameter scans at reasonable computing cost. Recently, a semi-Lagrangian simulation code, TERESA, has been developed based on this model, making efficient use of parallel computing [37 39]. However, given the limited system size adopted in our simulations to reduce computing costs, the frequency of modes can reach values comparable to the bounce-frequency of tungsten. Therefore, we check that the qualitative trends are still valid for lower- $Z$ impurities (carbon) for which the bounceaveraged treatment poses no such issue.

In section II], we describe the bounce-averaged gyrokinetic model, along with the boundary conditions, initial conditions, and our choice of input parameters. In section III, we analyze the impact of tungsten concentration on the linear growth rate. Further, we show how impurities impact the structure of the linear mode in energy-space. In section IV, we investigate the impact of tungsten concentration on turbulent intensity, and on radial density and heat fluxes. Figs. 9 and 10 are the two main figures of this paper. They show the limit of the passive treatment for transport studies. In section $\mathrm{V}$, we provide a summarizing figure and conclude. 


\section{MODEL}

We aim at modeling low-frequency (toroidal precession time-scale) turbulence in the core of a tokamak plasma composed of electrons, deuterium, and high- $Z$ tungsten impurities.

We adopt a reduced bounce-averaged gyrokinetic model, which was developed by Depret [33], Sarazin [34] and Darmet [35], based on the Tagger-Pellat-Diamond-Biglari model [31, 32], and has been recently applied to study impurity transport [28, 36]. The bounce-averaged gyrokinetic distribution $f_{s}$ of trapped particles (or 'banana centers') of species $s=D, W, e$ satisfies a kinetic equation,

$$
\frac{\partial f_{s}}{\partial t}+\left[J_{0} \phi, f_{s}\right]_{\alpha, \psi}+\omega_{d, s} \frac{\partial f_{s}}{\partial \alpha}=0
$$

Here, $\omega_{d, s}=E \Omega_{d} / Z_{s}$ is the energy-dependent precession frequency, $\Omega_{d}=\left(e R_{0}^{2} B_{\theta}\right)^{-1}, Z_{s}$ is the charge number of species $s\left(Z_{e}=-1, Z_{D}=1\right.$ and $\left.Z_{W}=40\right)$, and $[\ldots]_{\alpha, \psi}$ are Poisson brackets in the phase-space of toroidal precession angle $\alpha$ and poloidal magnetic flux $\psi$ (which plays the role of radial coordinate). Furthermore, $J_{0}$ is an operator which performs two successive averages: a gyro-average (on a cyclotron motion), and a bounce-average (on a banana orbit) [39]. The radial dependencies are neglected in this operator.

This reduced model relies on the following assumptions:

- The mode frequency is much lower than the bounce frequencies of trapped particles for all species. This assumption will be checked in Sec. III. It must be stressed that this assumption breaks down for heavy impurities since their bounce frequency decreases with the square root of their mass number. For our choice of parameters, some high-amplitude modes can reach frequencies of the order of the bounce frequency of tungsten. Therefore, these results can only be taken as qualitative trends, representative of cases with lower-mass impurities and larger system sizes than chosen here for tungsten. The carbon cases we analyze are well within the validity regime of the model.

- The equilibrium configuration is that of a large aspect ratio tokamak. However, the inverse aspect ratio $\epsilon$ must not be so small that the fraction $f_{p} \sim \sqrt{\epsilon}$ of trapped particles is negligible.

- Resonant interactions are dominated by strongly trapped particles. In this case we 
can neglect the radial variation of precession frequency, and we focus on a single value of the pitch-angle.

- The mode frequency is much lower than the passing particles transit frequency.

- The plasma is at low- $\beta$, for which trapped particles precession resonance-driven modes are mostly electrostatic.

Self-consistency is ensured by a quasi-neutrality constraint, including a polarization term $\bar{\Delta}_{s} \phi$, which involves a non-isotropic Laplacian operator,

$$
\bar{\Delta}_{s} \equiv\left(\frac{q_{0} \rho_{c, s}}{L_{\psi}}\right)^{2} \frac{\partial^{2}}{\partial \alpha^{2}}+\delta_{b, s}^{2} \frac{\partial^{2}}{\partial \psi^{2}} .
$$

Here, $L_{\psi}$ is the radial length of the simulation domain, $\rho_{c, s}$ is the Larmor radius, and $\delta_{b, s}$ is the banana width, all in units of $\psi$, and $q_{0}$ is a typical value of safety factor.

The quasi-neutrality equation reads

$$
\sum_{s} \frac{e Z_{s}^{2} n_{e q, s}}{T_{e q, s}}\left[\frac{1-f_{t}}{f_{t}}\left(\phi-\epsilon_{\phi, s}\langle\phi\rangle\right)-\bar{\Delta}_{s} \phi\right]=4 \pi \sqrt{2} \sum_{s} \frac{Z_{s}}{m_{s}^{3 / 2}} \int_{0}^{\infty} J_{0, s} f_{s} E^{1 / 2} \mathrm{~d} E,
$$

where $f_{t}$ is the fraction of trapped particles. The average $\langle\phi\rangle$ is an average on the angle $\alpha$.

In this reduced model, passing particles are treated quasi-adiabatically. The free parameters $\epsilon_{\phi, s}$ controls the response of passing particles to electric perturbations. For passing electrons, we impose $\epsilon_{\phi, e}=1$, consistently with their lack of response to the zonal potential in the limit of electron gyro-radius much smaller than the characteristic radial variation of zonal flows [10]. For passing deuterium and tungsten, which do respond to zonal flows, we impose $\left(\epsilon_{\phi, D}=\epsilon_{\phi, W}=0\right)$.

In this paper, the TERESA simulations are performed with thermal baths at both outside boundary $\psi=0$ and inside boundary $\psi=L_{\psi}$, and without any source nor sink. Note that in our model, $\psi$ is not the true poloidal magnetic flux which is roughly proportional to $-r^{2}$, but a shifted version of it, such that the magnetic axis $r=0$ corresponds to $\psi=L_{\psi}$, and the outer radial boundary corresponds to $\psi=0$. Artificial dissipation is imposed in buffer regions $\psi<0.15 L_{\psi}$ and $\psi>0.85 L_{\psi}$ to smooth out the transition between turbulent fluctuations $\phi$, and the constraint $\phi=0$ at $\psi=0$ and $\psi=L_{\psi}$.

Throughout this paper, the equilibrium distribution functions are chosen as

$$
f_{\mathrm{eq}, s}(\psi, E)=\frac{n_{\mathrm{eq}, s}}{\left(2 \pi T_{\mathrm{eq}, s} / m_{s}\right)^{3 / 2}} \exp \left(-\frac{E}{T_{\mathrm{eq}, s}}\right) .
$$




\begin{tabular}{|c|c|c|c|c|c|}
\cline { 2 - 4 } & $a / R_{0}$ & $f_{t}$ \\
\cline { 2 - 5 } 0.1 & $2 / 3$ & $C_{s}$ & $\tau_{s}$ \\
\hline Species $s$ & $Z_{s}$ & $\rho_{c, s} / L_{\psi}$ & $\delta_{b, s} / L_{\psi}$ & 1 & 1 \\
\hline $\mathrm{e}$ & -1 & 0.01 & 0.01 & 1 & 1 \\
$\mathrm{D}$ & 1 & 0.03 & 0.1 & $1-Z_{W} C_{W}$ & 1 \\
$\mathrm{~W}$ & 40 & 0.0072 & 0.024 & $10^{-6}$ to $10^{-2}$ & 1 \\
\hline
\end{tabular}

TABLE I. Input parameters for both cases 1 and 2 . Here, $a / R_{0}$ is the aspect ratio.

\begin{tabular}{|c|c|c|}
\hline & $\kappa_{n}$ & $\kappa_{T}$ \\
\hline Case 1 & $\mathbf{0 . 2 5}$ & $\mathbf{0 . 2 5}$ \\
\hline Case 2 & $\mathbf{0}$ & $\mathbf{0 . 5}$ \\
\hline
\end{tabular}

TABLE II. Input density and temperature gradients.

This is an approximation of a 2D Maxwellian where the radial dependency of the equilibrium Hamiltonian is neglected. This implies that curvature pinch is neglected, and that global effects due to the radial dependency of the precession frequency are neglected.

The initial radial density and temperature profiles for $0.2<\psi / L_{\psi}<0.8$ are linear in $\psi$,

$$
\begin{aligned}
& n_{\mathrm{eq}, s}(\psi)=n_{0, s}\left(1+\kappa_{n} \frac{\psi}{L_{\psi}}\right), \\
& T_{\mathrm{eq}, s}(\psi)=T_{0, s}\left(1+\kappa_{T} \frac{\psi}{L_{\psi}}\right),
\end{aligned}
$$

where the equilibrium densities at $\psi=0, n_{0, s}$, are such that $\sum_{s} Z_{s} n_{0, s}=0$, and where $\kappa_{n}$ and $\kappa_{T}$ are species-independent input parameters, which measure the equilibrium radial gradients in terms of $\psi$ (therefore, positive $\kappa_{n, T}$ correspond to typical negative gradients in $r$ ). Note that this choice is different from that of Refs. [33, 39 41], which adopted a first-order Taylor expansion in $\psi$. For $\psi / L_{\psi}<0.2$ or $>0.8$, the equilibrium gradients are gradually diminished toward zero at the boundaries $\psi=0$ and $\psi=L_{\psi}$ (as shown in Fig. 6). This is done to avoid instabilities at these boundaries, consistently with our boundary condition $\phi=0$.

The density and temperature ratios between species are noted as $C_{s}=n_{0, s} / n_{0, e}$, and $\tau_{s}=T_{0, i} / T_{0, s}$. 
In this paper, we analyze a series of TERESA simulations with the input parameters of Table I] for the two cases of Table II] and various values of tungsten concentrations $C_{W}$. Case 1 has equal density and temperature gradients, while case 2 has a flat density profile but a stronger temperature gradient. In all cases, we choose $n_{0, e}=n_{0}$, and $T_{0, e}=T_{0, D}=$ $T_{0, W}=T_{0}$, where $n_{0}$ and $T_{0}$ are used as arbitrary normalization constants. Therefore, $n_{0, W}=C_{W} n_{0}$, and $n_{0, D}=\left(1-Z_{W} C_{W}\right) n_{0}$ to satisfy quasineutrality. The ratios $\rho_{c, W} / \rho_{c, D}$ and $\delta_{b, W} / \delta_{b, D}$ correspond to actual tungsten to deuterium mass ratio $\left(m_{W} / m_{D}=184 / 2\right)$. However, in order to limit the total cost of numerical resources, simulations are performed with artificially heavy electrons. The ratio $\rho_{c, e} / \rho_{c, D}$ corresponds to $m_{e} / m_{D}=1 / 9$ and the ratio $\delta_{b, e} / \delta_{b, D}$ corresponds to $m_{e} / m_{D}=1 / 100$. To have a self-consistent $m_{e} / m_{i}$ ratio would require a value of $\rho_{c, e} / L_{\psi}$ of 0.003 instead of 0.01 , which turns out to require quadrupling the computing cost to avoid numerical instabilities, and only has negligible impacts on our results. Furthermore, based on a few simulations with real electron mass, it appears that the impact of our hypothesis of heavy electrons is negligible. This was expected from the linear dispersion relation, where the electron mass only appears as terms of order $\left(n \rho_{c, e} / L_{\psi}\right)^{2}$ and $\left(k_{\psi} \delta_{b, e} / L_{\psi}\right)^{2}$ (both $\sim 10^{-2}$ for real electron mass and typical wave numbers).

The normalization in the simulation code is such that there is no need to choose a particular value for reference density $n_{0}$, temperature $T_{0}$, magnetic field strength, and safety factor profile $q(\psi)$. The results are obtained and shown as dimensionless quantities, for example: the potential as $e \phi / T_{0}$, and growth-rates as $\gamma / \omega_{0}$, where $\omega_{0}=\Omega_{d} T_{0}$ (the precession frequency of particles with velocity equal to the Deuterium thermal speed).

For these simulations, we use $N_{\psi}=513$ grid points in radius, $N_{\alpha}=513$ grid points in precession angle, and $N_{E}=128$ grid points in energy, with an energy cut-off $E_{\max }=20$.

For case 2, we observe that the direction of propagation is in the negative $\alpha$ direction, which is consistent with TEM instabilities dominating, in both linear and nonlinear phases. For case 1, the direction of propagation indicates a transition from TIM to TEM as the impurity concentration increases, with a transition concentration $C_{W, \operatorname{tr}} \approx 7 \times 10^{-4}$.

\section{LINEAR ANALYSIS}

For the purpose of this paper, we have developed a linear version of the TERESA code, which we have verified in two ways: 1 . against numerical solutions of the analytic dispersion 


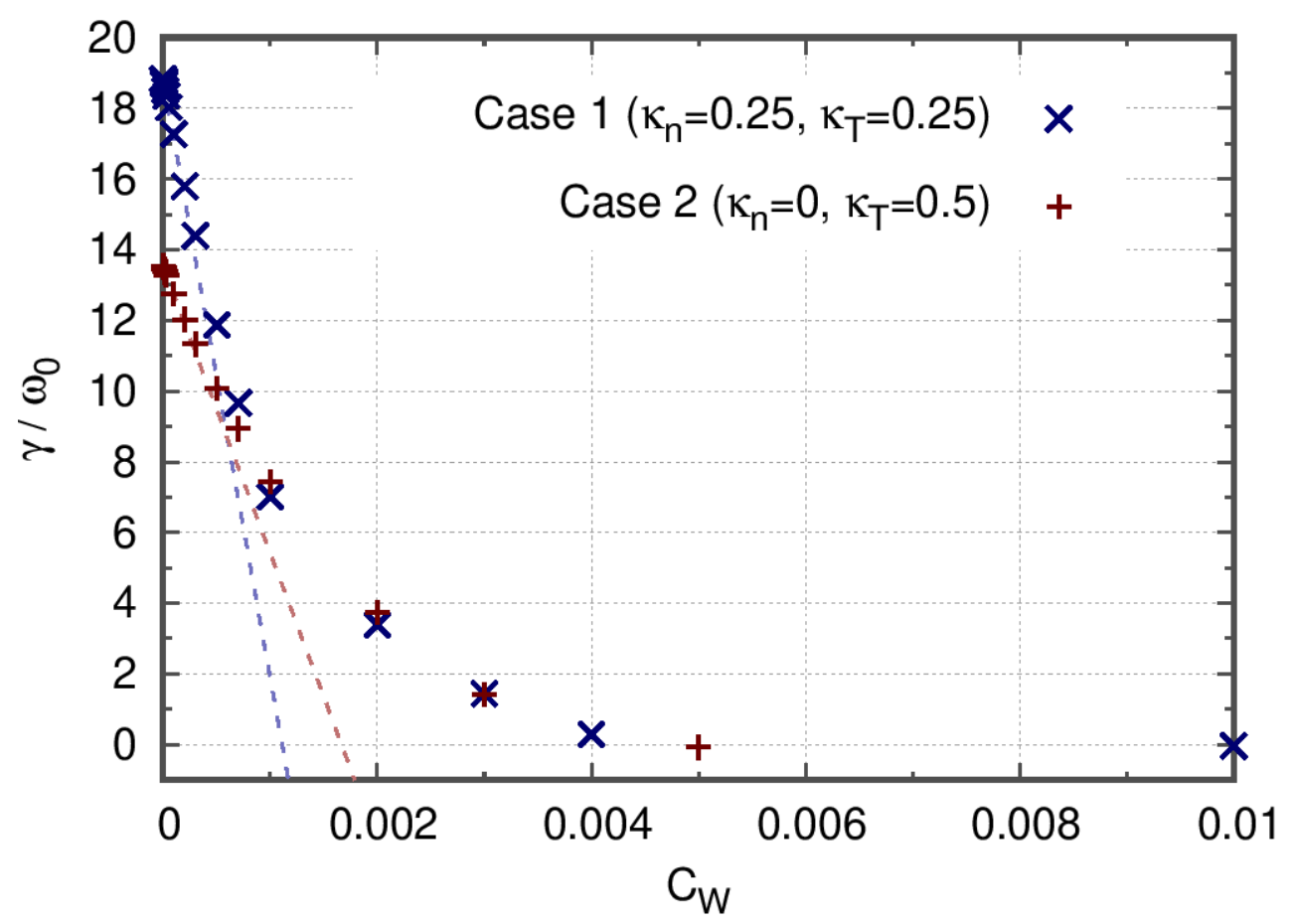

FIG. 1. Linear growth rate of the most unstable mode as a function of tungsten concentration. Two dashed lines show linear fits in the limit of vanishing concentrations, $\gamma=\gamma_{0}-a \omega_{0} C_{W}$, where $a=1.7 \times 10^{4}$ for case 1 , and $a=8.1 \times 10^{3}$ for case 2

relation, which was obtained in the limit of vanishing gradients, and 2. against single-mode simulations with the nonlinear version of TERESA.

\section{A. Growth rate}

Fig. 1 shows the linear growthrate $\gamma$ of the most unstable mode, as a function of tungsten concentration $C_{W}$. The growthrate decreases monotonously with $C_{W}$, consistently with Refs. [26 28, 42, 43], where impurities were found to have a stabilizing effect on trapped particle modes. In the literature, the stabilizing effect on ion modes is often interpreted as an effect of dilution of the ion density by the impurity. However, dilution does not affect electron density and thus does not directly accounts for the stabilizing effect on TEM. Based on our analysis of the linear dispersion relation, it appears that the stabilizing effect on TEM is mainly due a modification of the response of passing particles, at least for heavy impurity and low enough concentrations, consistently with Ref. [28]. Indeed, the coefficient in front 


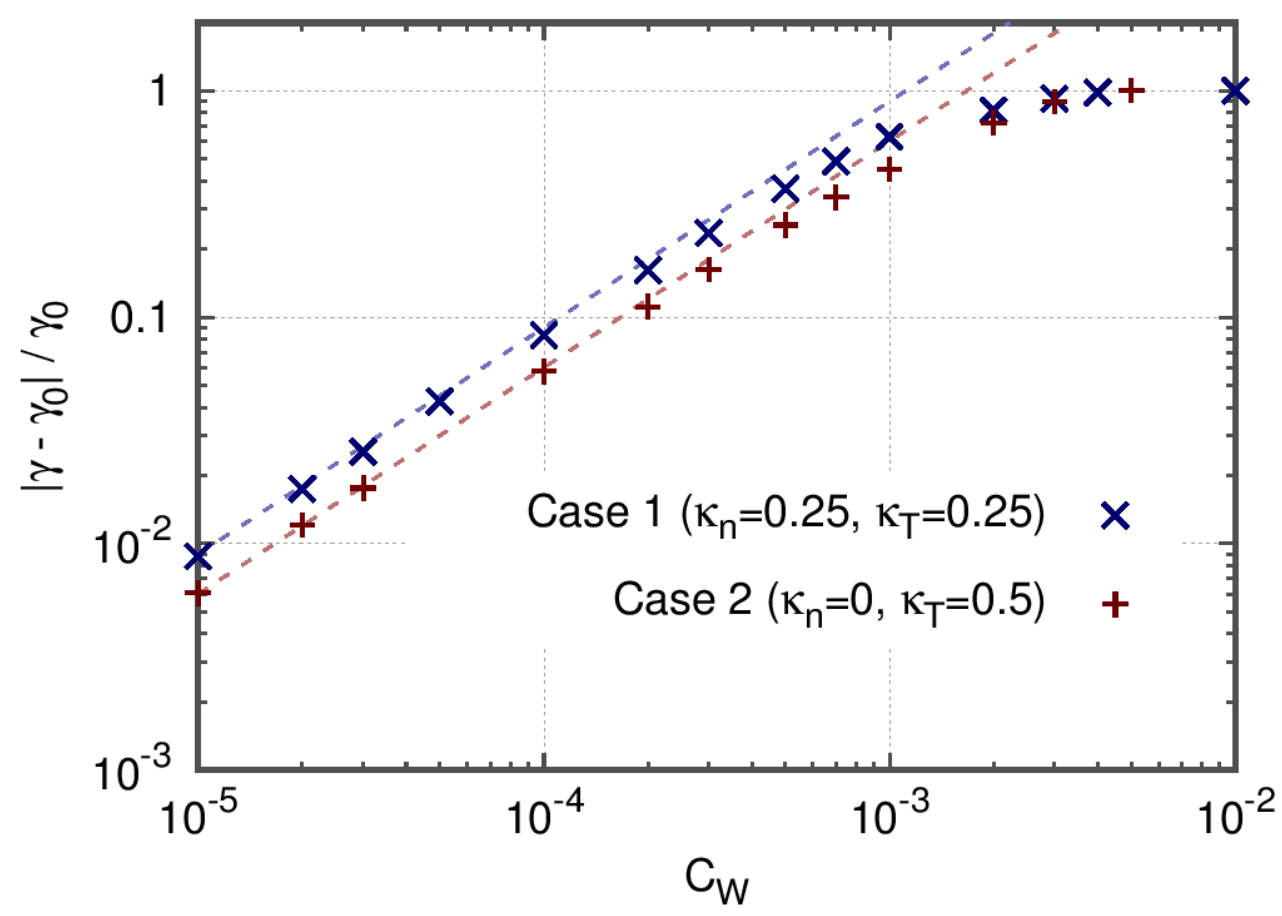

FIG. 2. Impact of tungsten concentration on the linear growth rate of the most unstable mode, in logarithmic scale. The dashed lines correspond to the same fit as in Fig. 1.

of $\phi$ in quasineutrality Eq. (3) is proportional to $\sum_{s} Z_{s}^{2} n_{0, s}$, which is roughly proportional to $2+Z_{W}^{2} C_{W}$. Therefore, heavy impurities can strongly affect the response of passing particles.

In the limit of vanishing concentration, $\gamma$ depends linearly on $C_{W}$. This behavior is better demonstrated in Fig. 2, which shows, in log-log scale, the relative impact of $C_{W}$ on the growth rate, $\left|\gamma-\gamma_{0}\right| / \gamma_{0}$, where $\gamma_{0}$ is defined as the growth rate in the absence of impurities.

A simulation where impurities are treated as a passive species yields $\gamma_{0}$, because noimpurity and impurities-treated-as-passive are the same from the point-of-view of quasineutrality. Therefore, the relative impact $\left|\gamma\left(C_{W}\right)-\gamma_{0}\right| / \gamma_{0}$ is also the relative error incurred by the passive treatment. We observe, for example, that the error is below $10 \%$ for concentrations below $10^{-4}$, and above $30 \%$ for concentrations above $10^{-3}$. Therefore, $W^{40+}$ tungsten cannot be treated as passive for $C_{W} \sim 10^{-3}$, consistently with [28]. Similar conclusions were found from gyrokinetic (GENE) simulations of light impurities in a JET-like plasma [29]. Note that $C_{W} \sim 10^{-3}$ corresponds to $C_{W} Z_{W}^{2} \sim 1$, at which point the contributions of tungsten and deuterium in the quasineutrality equation, Eq. (3), become comparable in 
amplitude.

The impact of impurities on the linear growth rate in the limit of low impurity concentrations can be estimated analytically. We write the dispersion relation formally as $D\left[\omega\left(C_{W}\right), C_{W}\right]=0$, where $\omega=\omega_{r}+\imath \gamma$ is the complex frequency. Then, to the first order in $C_{W}$, the dependency of the complex frequency on tungsten concentration, $\beta=$ $\mathrm{d} \omega /\left.\mathrm{d} C_{W}\right|_{C_{W} \rightarrow 0}$, is obtained from the following relationship,

$$
\left.\frac{\mathrm{d} D}{\mathrm{~d} C_{W}}\right|_{C_{W}=0}=\left.\frac{\partial D}{\partial C_{W}}\right|_{C_{W}=0}+\left.\beta \frac{\partial D}{\partial \omega}\right|_{C_{W}=0}=0 .
$$

The partial derivatives in Eq. (7) are calculated in Appendix $\|$ to the first order in $\left(\pi \delta_{b, D}\right)^{2}$, $\left(m \rho_{c, D}\right)^{2},\left(\pi \delta_{b, W}\right)^{2}$ and $\left(m \rho_{c, W}\right)^{2}$, and zeroth order in $\left(\pi \delta_{b, e}\right)^{2}$ and $\left(m \rho_{c, e}\right)^{2}$. This procedure yields $\gamma=\gamma_{0}-a \omega_{0} C_{W}$, with $a=2.2 \times 10^{4}$ for case 1 , and $a=8.9 \times 10^{3}$ for case 2 , which is in qualitative agreement with the linear fits shown in figures 1 and 2 .

Since the behavior is linear for $\left|\gamma-\gamma_{0}\right| / \gamma_{0}<0.3$, there is no clear threshold for the validity of the passive approach, as far as the linear growth rate is concerned. This is very unlike the case of radial fluxes of impurities, as will be demonstrated in section IV.

\section{B. Linear mode structure}

We investigate the structure of the linear mode. The eigenfunction is obtained from linear, single-mode TERESA simulations by extracting at a fixed time $t_{1}$ a single $m$ Fourier component of $f\left(\alpha, \psi, E, t_{1}\right)$. The time of snapshot $t_{1}$ is chosen such that the eigenfunction had ample time to form from the initial arbitrary perturbation. The radial structure is $\sin \left(\pi \psi / L_{\psi}\right)$, consistently with linear theory, which yields much larger growth rates for the larger radial wave compatible with boundary conditions $\phi=0$.

Let us now describe the structure of the linear mode in energy space, at a given radial location $\psi=\psi_{0}$. We choose $\psi_{0}=0.4 L_{\psi}$, near the mid-radius of the simulation box. We

note $\hat{\phi}_{m}$ the $m$-th Fourier component in $\alpha$ of the potential, and similarly $\hat{f}_{s, m}$ for $f_{s}$. Fig. 3 shows the absolute value of the eigenfunctions $\hat{f}_{s, m} / \hat{\phi}_{m}$, for the most unstable mode $m$. We compare two cases, without $\left(C_{W}=0\right.$, left $)$ and with $\left(C_{W}=10^{-3}\right.$, right $)$ impurities. In both cases, the eigenfunction is composed of a first component at $E / T_{0} \ll 1$, and a second component, which peaks at $E \approx E_{p}$, where $E_{p}$ is defined as the energy for which $\partial f_{\text {eq }} / \partial \psi$ is 

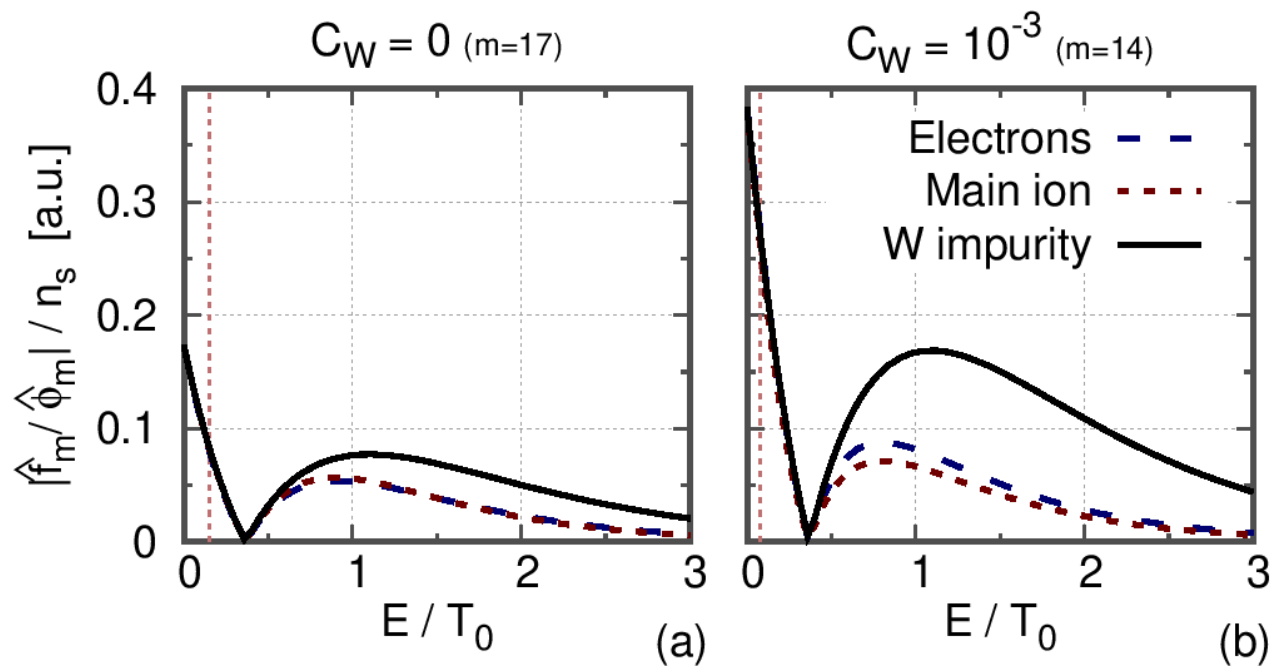

FIG. 3. Eigenfunction for Case 1, at $\psi=\psi_{0}$. (a) Passive treatment of impurities. (b) Active treatment with $C_{W}=10^{-3}$. Each dotted vertical line corresponds to the energy such that the resonance condition $\left|\omega_{r}\right|=m \Omega_{d} E$, is satisfied.

maximum. We have $E_{p} / T_{0}=5 / 3-2 \kappa_{n} /\left(3 \kappa_{T}\right)=1$ in the present case.

In the limit of small $\rho_{c}$ and $\delta_{b}$, the eigenfunction can be estimated as

$$
\hat{f}_{m, s}(\psi, E)=\frac{m \partial f_{\mathrm{eq}, s} / \partial \psi}{\omega_{r}-m \Omega_{d} E / Z_{s}+\imath \gamma} \hat{\phi}_{m}(\psi)
$$

The latter expression is in quantitative agreement with the values from numerical simulation. Note that, since $\gamma_{r}>\omega$, the peak near $E=E_{p}$ is relatively far from the resonant energy $E_{r, s}=Z_{s} \omega_{r} /\left(m \Omega_{d}\right)$.

As can be seen in Eq. (8), the normalized distribution function $\hat{f}_{m, s} / n_{s}$ does not depend explicitly on $C_{W}$. In fact, here, the impact of impurities on the shape of the eigenfunction comes mostly from the difference in growth rate, which is $\gamma=18.8 \omega_{0}$ for $C_{W}=0$ and $\gamma=7.01 \omega_{0}$ for $C_{W}=10^{-3}$. The frequencies are $\omega=2.52 \omega_{0}$ for $C_{W}=0$ and $\omega=-1.11 \omega_{0}$ for $C_{W}=10^{-3}$.

Let us now check our initial assumption that the mode frequency is much lower than the bounce frequencies of trapped particles for all species. Since the typical mode frequency is of order of the precession frequency for thermal deuterium, $\omega \sim \omega_{0}$, its ratio with the 
energy-dependent bounce frequencies $\omega_{b, s}(E) \approx\left(\epsilon E / m_{s}\right)^{1 / 2} /\left(q_{0} R_{0}\right)$ is

$$
\frac{\omega}{\omega_{b, s}} \sim\left(\frac{m_{s}}{m_{D}} \frac{T_{0}}{E}\right)^{1 / 2} \frac{q_{0}^{2}}{(2 \epsilon)^{1 / 2}} \frac{\rho_{c, D}}{a} .
$$

With our parameters, $\omega / \omega_{b, e} \sim 0.007\left(T_{0} / E\right)^{1 / 2}$ and $\omega / \omega_{b, D} \sim 0.07\left(T_{0} / E\right)^{1 / 2}$, which are both much below unity, even for deuterium particles at their small resonance energy $E_{r, D} \approx$ $0.37 T_{0}$. As for tungsten, $\omega_{d, W} / \omega_{b, W} \sim 0.016\left(T_{0} / E\right)^{1 / 2}$, but $\omega / \omega_{b, W} \sim 0.6\left(T_{0} / E\right)^{1 / 2}$, therefore some of the modes can reach a frequency comparable to the bounce-frequency of thermal tungsten particles. For this reason, the current model with the current parameters may miss some important effect of turbulence on tungsten transport. However, the ratio $\omega / \omega_{b, W}$ is proportional to $\rho^{*}=\rho_{c, D} / a$, which is 0.03 here. For lower values of $\rho^{*}$, the model regains its validity. Similarly, the ratio $\omega / \omega_{b, W}$ is proportional to $m_{s}^{1 / 2}$, so the model regains its validity for lower-mass impurities. Finally, the results presented in this paper for tungsten are to be taken as qualitative trends, which are only representative of cases with lower $\rho^{*}$ and/or lower-mass impurities.

\section{NONLINEAR ANALYSIS}

\section{A. Turbulence}

Let us first consider a typical case, namely case 1 with $C_{W}=10^{-5}$, before varying the parameters. Fig. 4 shows the time-evolution of the electric potential at $\psi=\psi_{0}$. The modes $m>30$ stay at low amplitudes $\left(e\left|\hat{\phi}_{m}\right| / T_{0}<3 \times 10^{-3}\right)$, and are not represented. Each mode for $m \leq 30$ is represented, and we emphasize the most unstable mode $m=17$. We measure the strength of turbulence via the root mean square (rms) of the non-zonal potential, $\phi_{\text {rms }}$,

where $\phi_{\mathrm{rms}}^{2}=\left\langle(\phi-\langle\phi\rangle)^{2}\right\rangle$, and averages are over $\alpha$. In the steady-state, the amplitude of the zonal mode $m=0$ is much lower than the rms of the non-zonal potential.

We observe the formation of TIM turbulence after the linear phase. The turbulent field reaches a quasi-steady-state after a time of the order of a few $\omega_{0}^{-1}$. We recall that $\omega_{0}=T_{0, D} \Omega_{d}$ is the precession frequency of thermal deuterium.

Fig. 5 shows how the turbulence strength in the quasi-steady-state depends on the impurity concentration, for case 1. Increasing impurities weakens turbulence. The impact is linear for $C_{W}<6 \times 10^{-4}$. This trend is in qualitative agreement with how the growth 


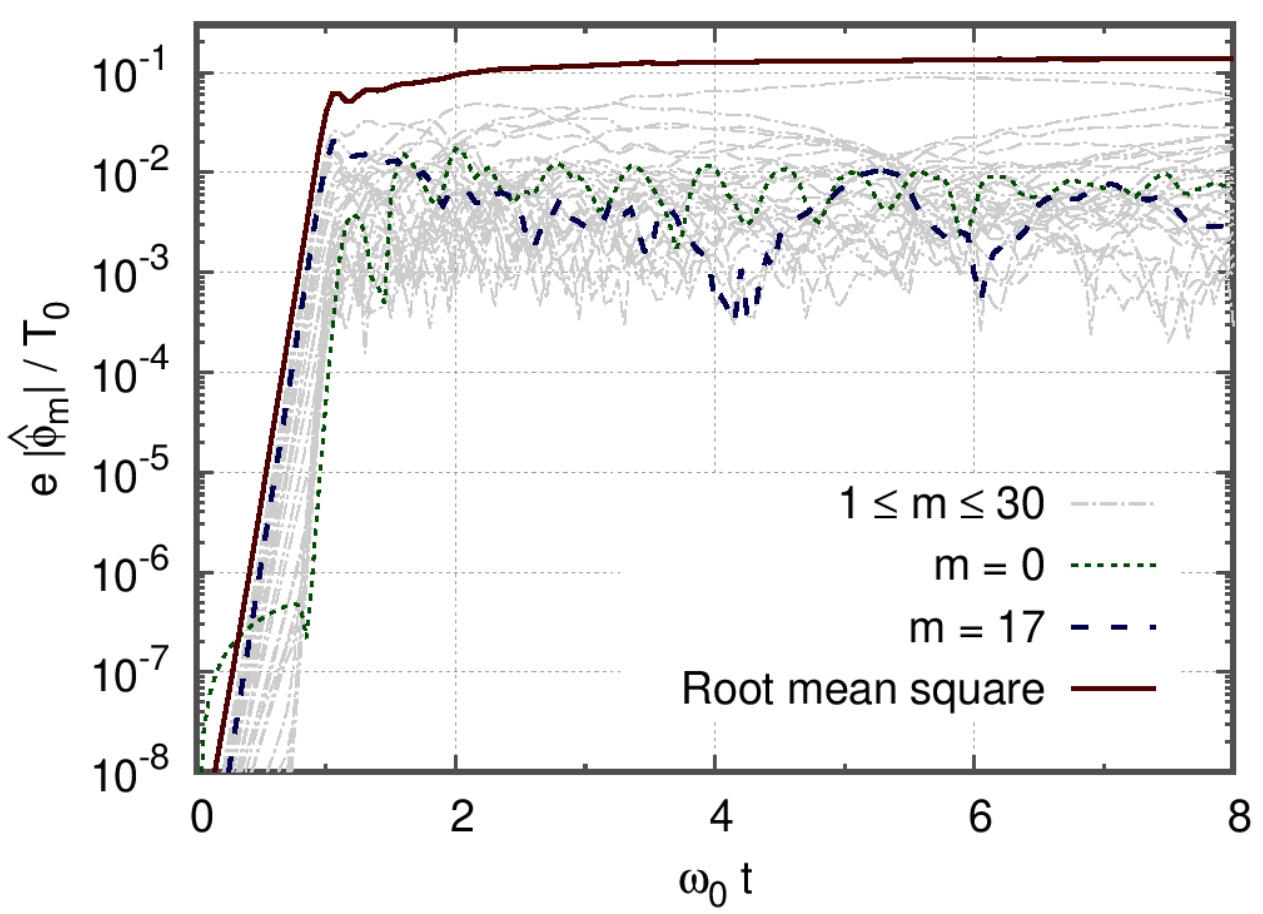

FIG. 4. Time evolution of the electric potential at $\psi=\psi_{0}$ for Case 1 , with $C_{W}=10^{-5}$.

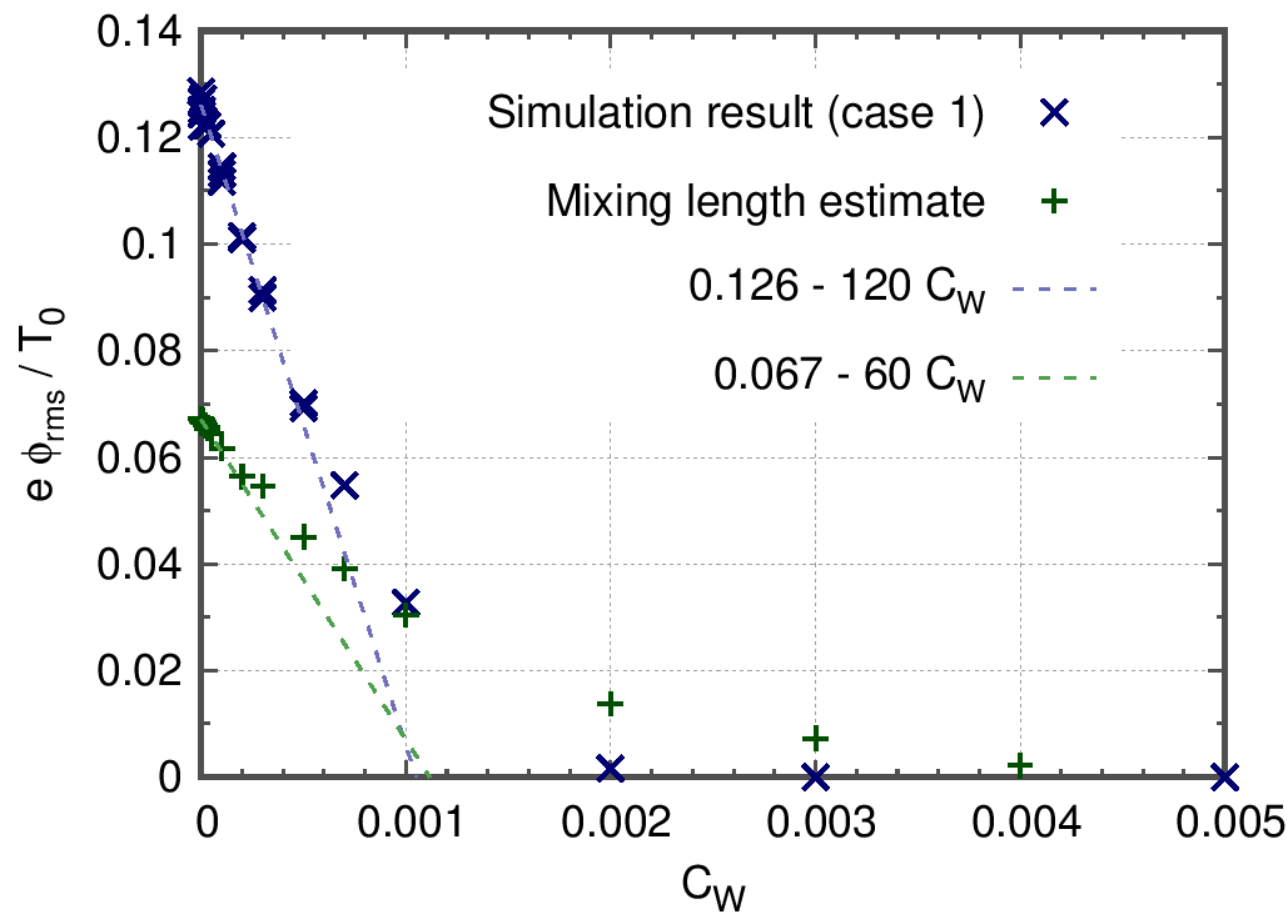

FIG. 5. Time-averaged electric potential as a function of tungsten concentration for Case 1. Dashed lines are linear fits as $C_{W} \rightarrow 0$. 
rate depends on tungsten concentration. However, the potential is not simply proportional to the growth rate. This can be seen in Fig. 5, which includes a mixing length estimate, $e \phi / T_{0}=\gamma /\left(k_{r} \rho_{c, i} k_{\theta} c_{s}\right)$. Although there is a rough order-of-magnitude agreement, the slope of the linear fit of the potential as $C_{W} \rightarrow 0$, is twice that of the mixing length estimate.

Since $e \phi_{\mathrm{rms}} / T_{0} \approx 0.126-120 C_{W}$ (for $\left.C_{W}<6 \times 10^{-4}\right)$, there is no clear threshold for the limit of validity of the passive approach, but one can conclude for example that a passive treatment yields a relative error $<10 \%$ for $C_{W}<10^{-4}$, and $>30 \%$ for $C_{W}>3 \times 10^{-4}$. For $C_{W} \approx 2 \times 10^{-3}$ the rms of the potential is reduced by almost 2 orders-of-magnitude compared to $C_{W}=0$.

The main message here is that a linear analysis is not enough to conclude about the regime of validity of the passive treatment of impurities for the turbulence intensity.

\section{B. Particle transport}

Let us first consider the same typical case as in subsection IV A, namely case 1 with $C_{W}=10^{-5}$, before varying the parameters.

Fig. 6 shows the radial profiles of density and temperature. The figure includes the initial profile $(t=0)$, as well as a snapshot of the profiles in the quasi-steady-state. We choose $\omega_{0} t=5$. Note that the densities are normalized to $n_{0, s}$, and one should keep in mind that in this case the tungsten density is $10^{5}$ lower than the electron and deuterium densities. In the quasi-steady-state, we observe that, for both density and temperature, while the electrons and deuterium maintain positive gradients, the tungsten profiles are completely flattened. These profiles do not evolve significantly for longer times (we have checked until $t \approx 30 \omega_{0}^{-1}$ ).

Fig. 7 illustrates how these radial profiles evolve in time, with different timescales. The figure shows the time-evolution of the densities and temperatures measured at $\psi=\psi_{0}=$ $0.4 L_{\psi}$, which is indicative of the time-evolution of the global profile. Note that although density and temperature increase at this location, it decreases at other locations such as $\psi=0.6 L_{\psi}$. Overall, total particle and energy conservation are ensured (with relative errors of $\sim 10^{-6}$ and $<1 \%$, respectively). After the linear phase, we observe that tungsten profiles are flattened within a very short time, $\sim 0.1 \omega_{0}^{-1}$. In contrast, electron and deuterium profiles evolve on a much longer timescale, $\approx 5 \omega_{0}^{-1}$. The results are qualitatively similar for other concentrations - with a strong flattening of the impurity density and temperature 

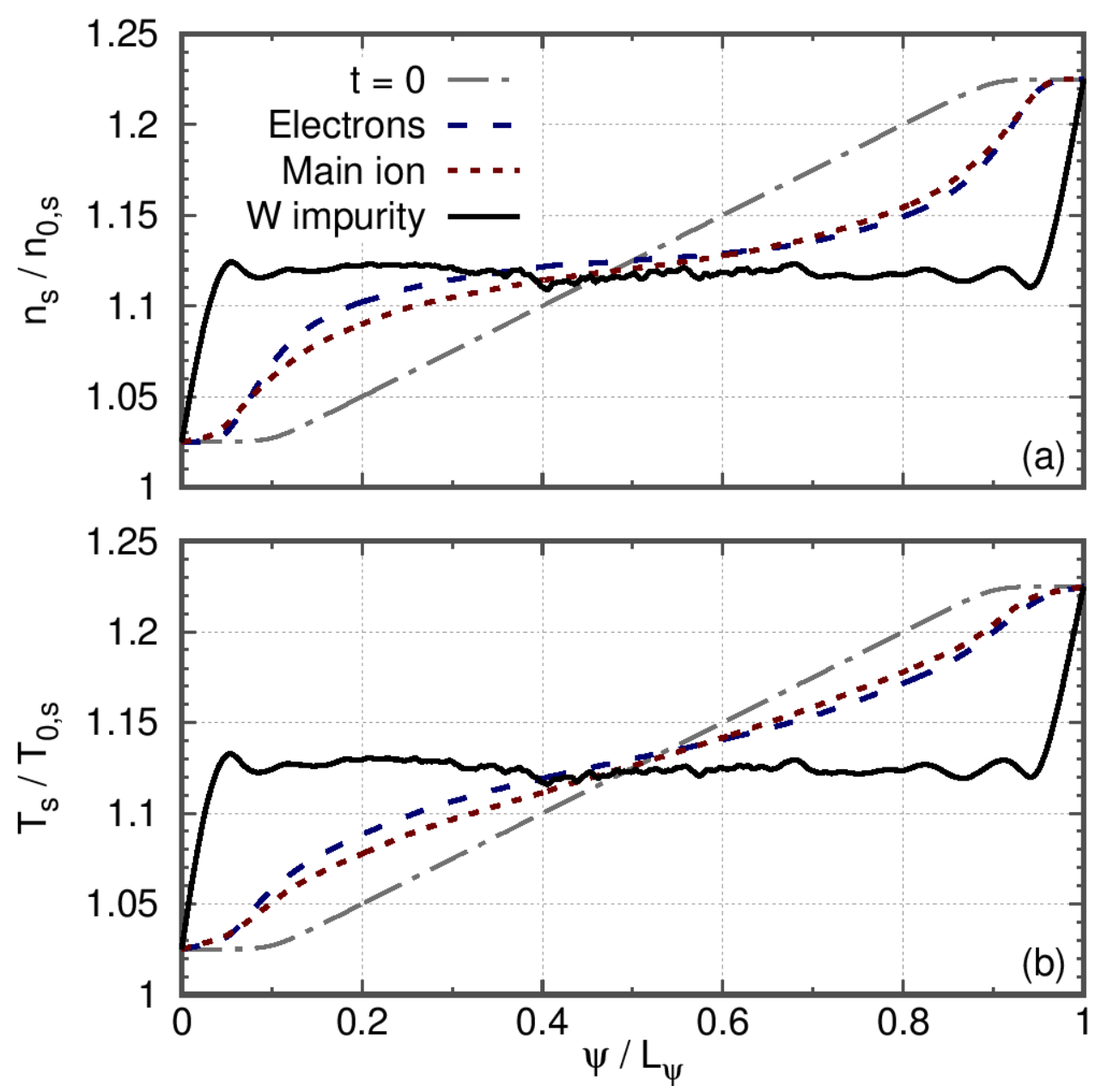

FIG. 6. Snapshots of the radial profiles of density (a) and temperature (b) for Case 1, with $C_{W}=10^{-5}$. A curve labeled $t=0$ shows the initial profiles, while the other curves are snapshots at $\omega_{0} t=5$.

profiles - except that, for high concentrations $\left(C_{W}>2 \times 10^{-3}\right)$, the timescale of flattening for impurities becomes of the same order as that for electrons and main ions $\left(\approx 5 \omega_{0}^{-1}\right)$.

These observations - including the strong flattening for the impurity - are consistent with a diffusion of pressure for all species, where the diffusion coefficient increases with increasing turbulence intensity, and where turbulence intensity decreases with decreasing electron and/or main ion gradients.

Finally, let us focus on radial particle flux (or density flux). The radial particle flux $\Gamma$ is defined such that, in the absence of source, the angle-averaged density satisfies

$$
\frac{\partial\langle n\rangle_{\alpha}}{\partial t}+\frac{\partial \Gamma}{\partial \psi}=0
$$



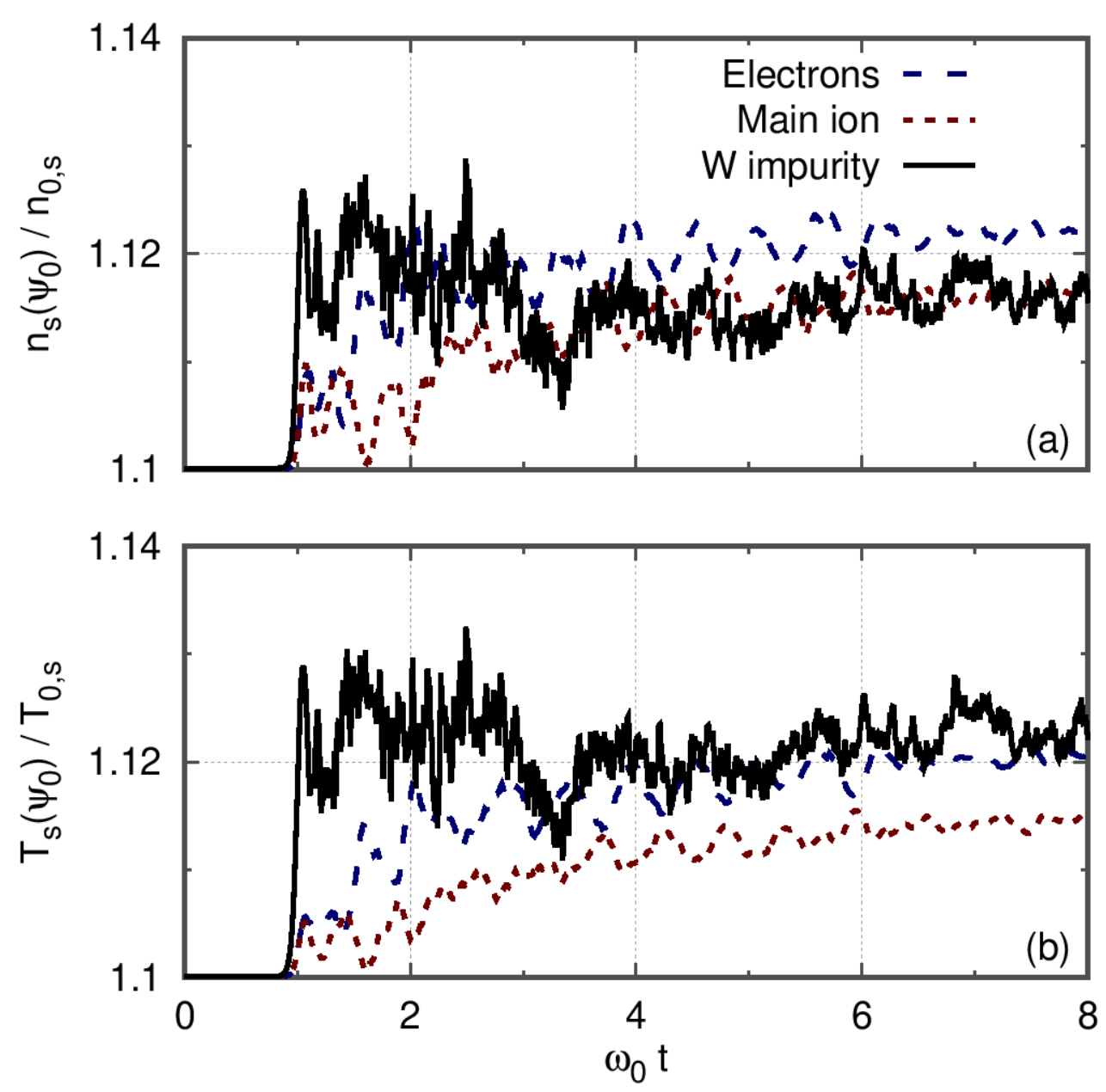

FIG. 7. Time-evolution of the density (a) and temperature (b) at $\psi=\psi_{0}$, for Case 1, with $C_{W}=10^{-5}$.

In the following figures, the particle flux is normalized to $\Gamma_{0, s}=\left(n_{0, s} / n_{0}\right) \Gamma_{0}$, where $\Gamma_{0}=$ $n_{0} \rho^{*} c_{s}, \rho^{*}=\rho_{i} / a$, and $c_{s}=\sqrt{\left(T_{e}+3 T_{i}\right) / m_{D}}$ is the ion sound speed. Note that $\Gamma_{0}$ is linked to a gyro-Bohm estimate $\Gamma_{g B}=\rho^{*} T_{i} /\left(e B_{0}\right) \partial n / \partial r$ as $\Gamma_{0} / \Gamma_{g B}=c_{s} / v_{*, i}$, where $v_{*, i}$ is the ion diamagnetic velocity. With this normalization, a positive normalized flux corresponds to a flux from the core toward the edge. To apply this normalization, we choose an aspect ratio $a / R_{0}=0.1$, and a safety factor $q(r)=1.2+3(r / a)^{2}$.

We focus on the flux at the given location $\psi=\psi_{0}$. In the quasi-steady state, $\Gamma\left(\psi_{0}, t\right)$ is a rapidly fluctuating function of time, with an average much smaller than the fluctuation amplitude. Therefore, it is appropriate to apply a moving average in time. To guide the choice of time-window, we compute the time-cumulative flux, $\int_{0}^{t} \Gamma\left(\psi_{0}, t^{\prime}\right) \mathrm{d} t^{\prime}$. Fig. 8 shows 


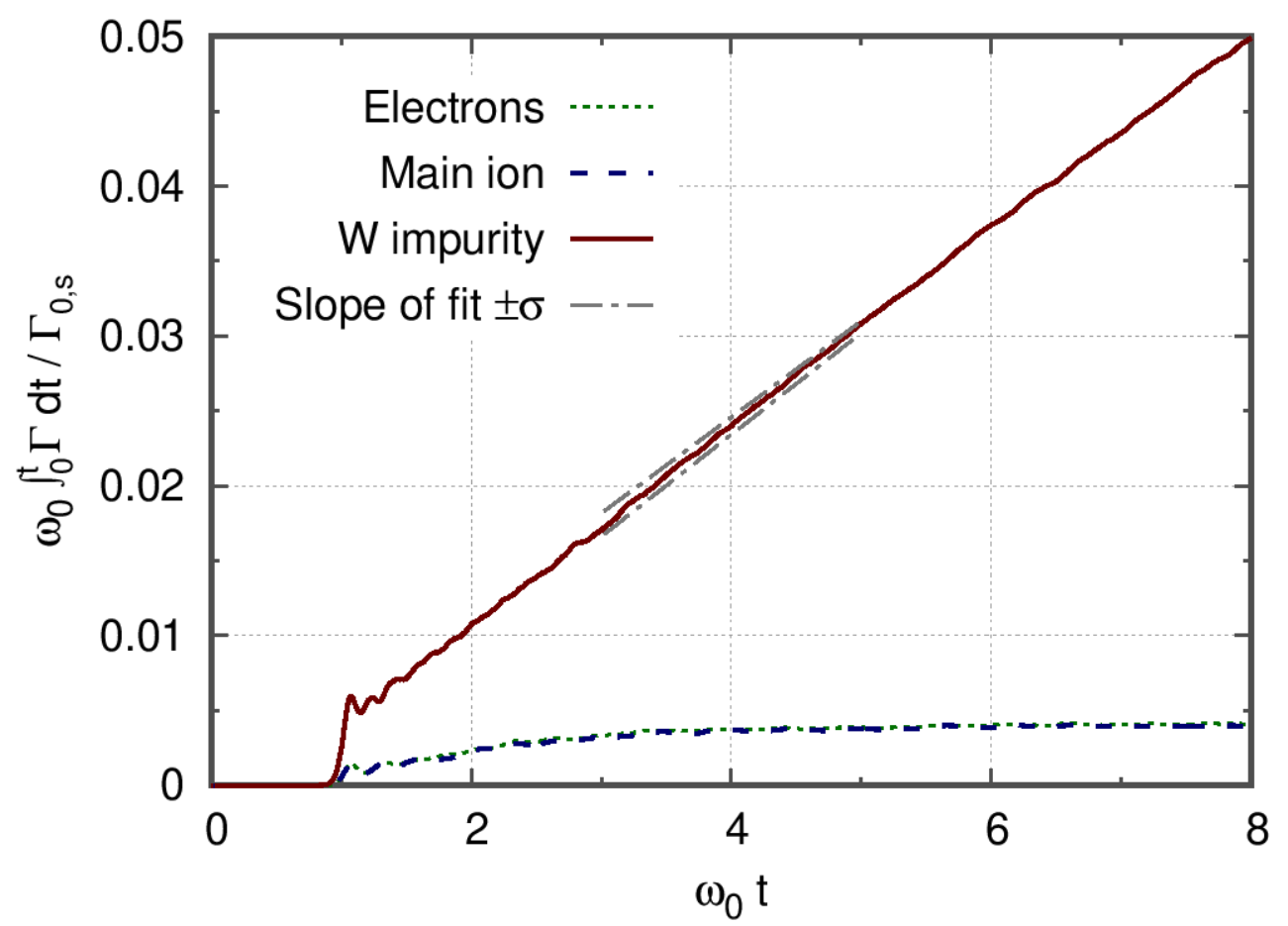

FIG. 8. Time-evolution of time-cumulative radial particle flux, at $\psi=\psi_{0}$, for Case 1 and $C_{W}=$ $10^{-5}$. Here, the flux is normalized to $\Gamma_{0}=\rho^{*} n_{0} c_{s}$.

the time-evolution of the time-cumulative radial particle fluxes for our reference case. We observe that fluxes are mostly positive, which is consistent with a flattening of initial density profiles. Normalized impurity transport is larger than the transport of electrons and main ions, but only because the normalization includes density. The non-normalized transport of impurities is actually orders-of-magnitude smaller than that of electrons and main ions. After the linear phase, impurity flux reaches a steady-state value, for $\omega_{0} t>1.5$, as indicated by the fact that the slope of the cumulative flux is roughly constant. Although we observe a similar behavior for different values of $C_{W}$, the timing of the linear phase varies. We choose a fixed timing of $3<\omega_{0} t<5$, which allows to capture the steady-state flux for all scanned values of $C_{W}$. We fit a line within this time interval which we use to extract the value of the steady-state impurity flux. Fig. 8 includes two line segments which represent the fit, plus or minus an uncertainty $\sigma$ due to the fluctuations. Since at the steady-state, the tungsten profiles are flattened, one should keep in mind that the instantaneous, local gradients can be completely different from the values of $\kappa_{n}$ and $\kappa_{T}$, which represent the initial gradients or the difference between boundary conditions. 


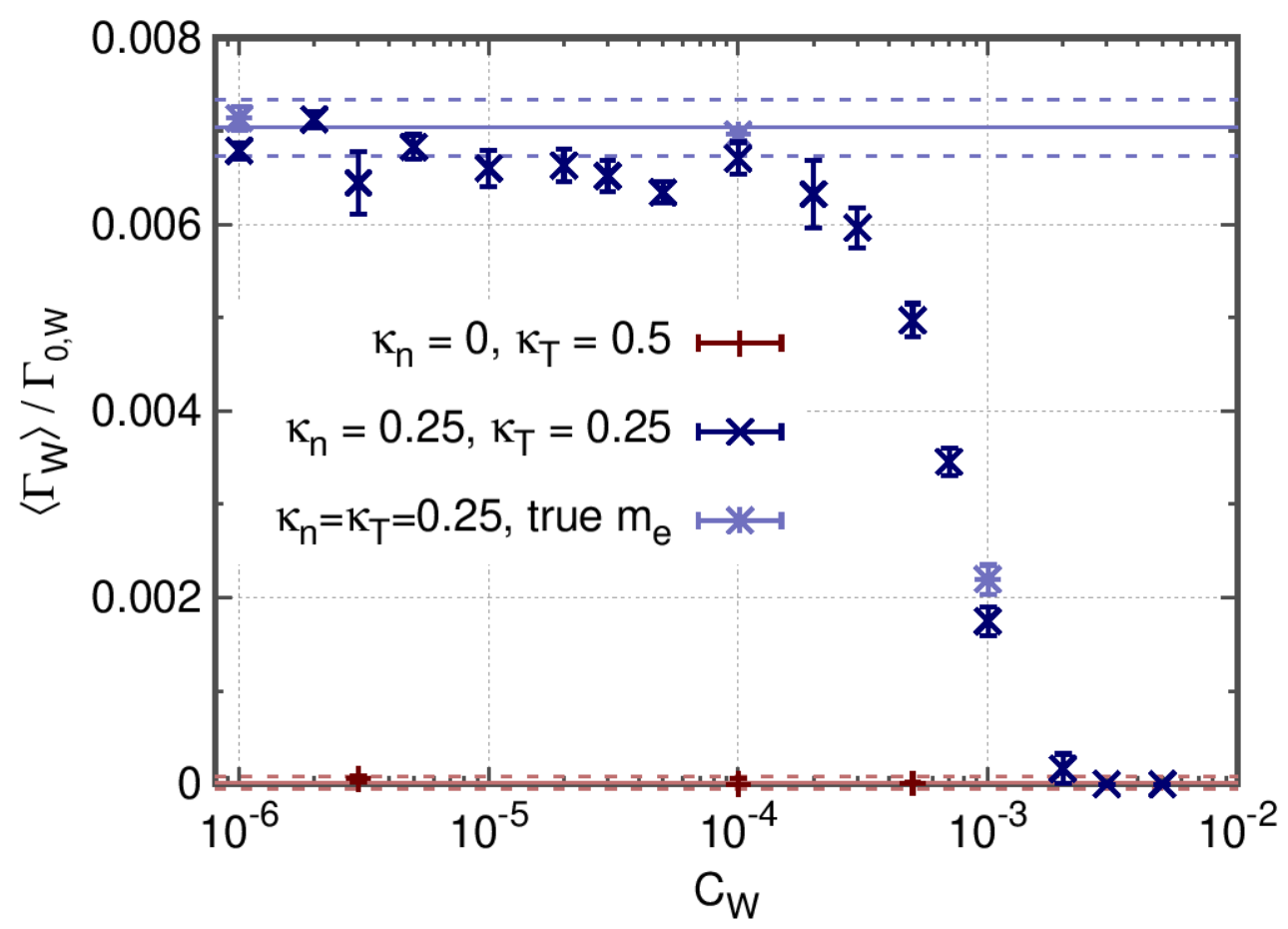

FIG. 9. Impact of tungsten concentration on the radial tungsten density flux, at $\psi=\psi_{0}$. The flux is normalized to $\Gamma_{0, W}=\rho^{*} n_{0, W} c_{s}$. Each point is an average between several TERESA simulation with active tungsten, and error bars represent the standard deviation $\sigma$. Horizontal lines correspond to the passive simulations (solid: average value, dashed: average $\pm \sigma$ ). Includes three simulations with true particle masses.

We repeat the above analysis for case 1 and case 2, and various values of tungsten concentration. For a given case and a given tungsten concentration, we perform between 2 and 10 simulations with different random phases of the initial perturbation, to take into account the effects of statistical fluctuations. In total, we performed and analyzed (via an automated process) 116 TERESA simulations. Fig. 9 shows how the tungsten density flux depends on tungsten concentration. Each point corresponds to a series of simulations (3 for most points) with tungsten treated as an active species, and the errorbar indicates the uncertainty $\sigma$, which originates from both statistical fluctuations and uncertainty in the linear fitting of the slope of cumulative flux. The horizontal lines show the same information but for the simulations where tungsten is treated as a passive species.

The density flux can be ignored for case 2, with its flat density profiles. Therefore we focus on case 1 . In contrast with the linear growth rate and the turbulence intensity, the 
density flux dependency on tungsten concentration is strongly nonlinear. There is a steep transition at $C_{W}=2 \times 10^{-4}$, below which the difference in flux between active simulations and passive simulations is not discernible from statistical fluctuations, and above which the passive treatment quickly yields large errors.

Although the model is meant as a tool to uncover trends, rather than to predict or interpret tokamak experiments quantitatively, let us nonetheless check whether the obtained fluxes are in the correct order-of-magnitude, keeping in mind that we had to assume a somewhat unrealistic aspect ratio of $a / R_{0}=0.1$. We compare our results to the flux at mid-radius $\Gamma_{W 40}^{\mathrm{XGa}} \approx 5 \times 10^{15} \mathrm{~m}^{-2} \mathrm{~s}^{-1}$ calculated in a XGCa simulation of a JET-like plasma with a concentration $C_{W}=2.5 \times 10^{-4}$ of $Z=40$ tungsten [44]. The density gradients are roughly similar to our setup, however, the temperature gradients. With $\rho * \sim 10^{-3}$, $n_{0, W} / C_{W} \sim n_{0, e} \sim 8 \times 10^{19} \mathrm{~m}^{-3}$, and $c_{s} \sim 3 \times 10^{5} \mathrm{~m} . \mathrm{s}^{-1}$, we obtain $\Gamma_{0, W} \sim 6 \times 10^{18} \mathrm{~m}^{-2} \cdot \mathrm{s}^{-1}$. Therefore, $\Gamma_{W 40}^{\text {TERESA }} \approx 36 \times 10^{15} \mathrm{~m}^{-2} \mathrm{~s}^{-1}$, which is 6 times higher than the value obtained in XGCa. However, this discrepancy appears to be mainly due to the aspect ratio $a / R_{0}=0.1$ chosen in our simulations. We performed one additional TERESA simulation, with a JETlike aspect ratio $a / R_{0}=0.4$. In this case we obtain $\Gamma_{W 40}^{\mathrm{TERESA}} \approx 12 \times 10^{15} \mathrm{~m}^{-2} \mathrm{~s}^{-1}$, which is in the correct order-of-magnitude.

\section{Energy transport}

The radial heat flux $q$ is defined such that, in the absence of source, the angle-averaged pressure satisfies

$$
\frac{\partial\langle n T\rangle_{\alpha}}{\partial t}+\frac{\partial q}{\partial \psi}=0
$$

We apply the same procedure to analyze the heat flux in the same 116 TERESA simulations. Fig. 10 shows how the tungsten heat flux depends on tungsten concentration. The heat flux is lower for the case 2 with steeper temperature profile but flat density profile, in qualitative agreement with the linear growth rates and turbulence intensities. In both cases 1 and 2, the heat flux dependency on tungsten concentration is strongly nonlinear. Again, this is in contrast with the linear growth rate and the turbulence intensity. From this figure, we conclude that the passive approach yields accurate estimates of the tungsten heat flux for concentrations below $C_{W}=2 \times 10^{-4}$ for case 1 , and below $C_{W}=10^{-4}$ for case 2 . 


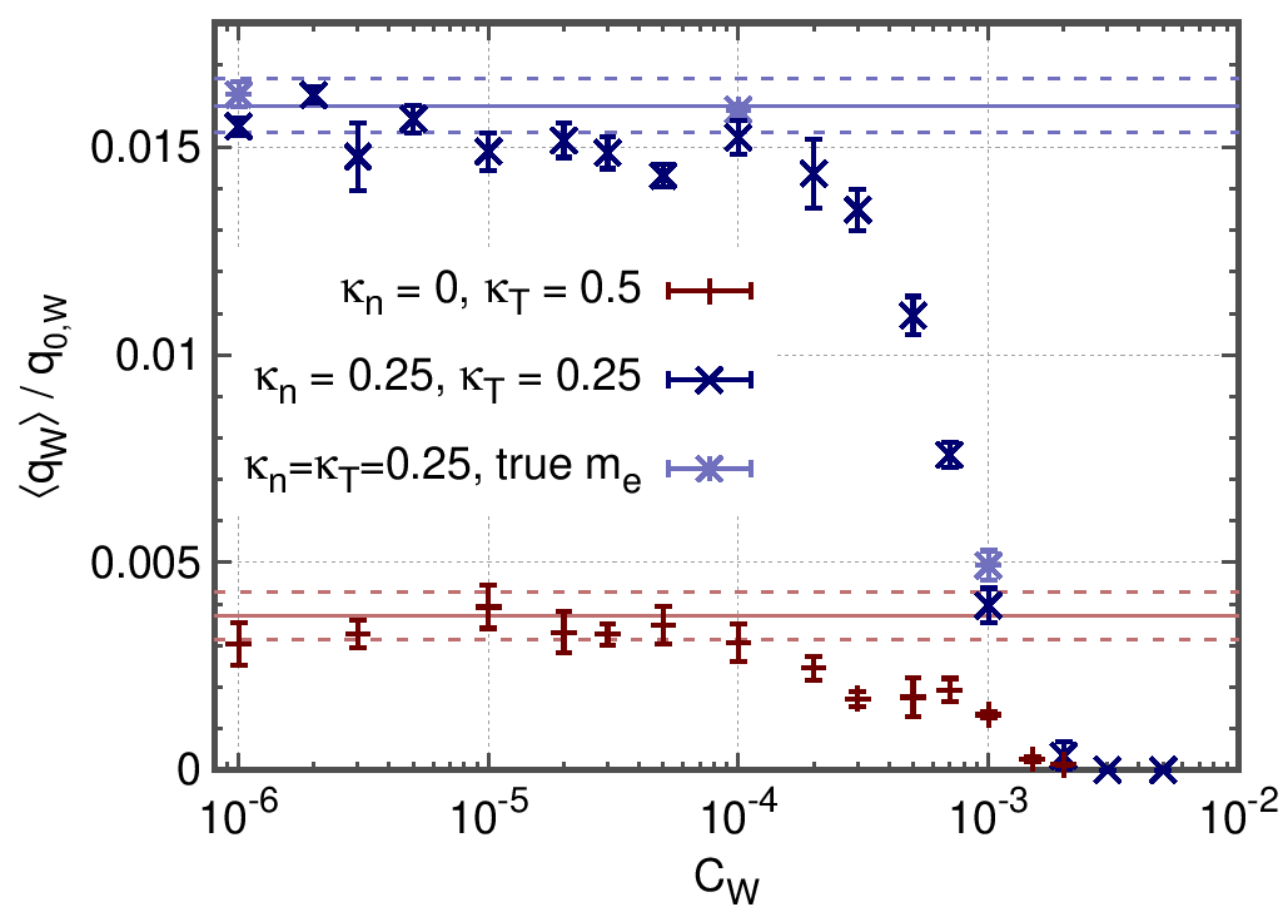

FIG. 10. Impact of tungsten concentration on the radial heat flux, at $\psi=\psi_{0}$. The flux is normalized to $q_{0, W}=\rho^{*} n_{0, W} T_{0} c_{s}$. Horizontal lines correspond to the passive simulations (solid: average value, dashed: average $\pm \sigma)$. Includes three simulations with true particle masses.

\section{Synchronisation of impurity density fluctuations}

Let us investigate the physical mechanism responsible for the steep transition between passive (trace) and active (non-trace) behavior of impurity transport. If we only consider the rms of fluctuations, the product $\phi_{\mathrm{rms}} n_{W, \mathrm{rms}}$ decreases with increasing tungsten concentration, which partly explains the reduction of density transport. However, the transition in terms of transport is significantly steeper. For example, between $C_{W}=10^{-4}$ and $C_{W}=10^{-3}$, the flux is reduced by a factor 3.9 , while the product $\phi_{\mathrm{rms}} n_{W, \mathrm{rms}}$ is only reduced by a factor 2.3 .

For a given mode $m$, we denote $\Phi_{n, W}$ the phase of tungsten density fluctuation, and $\Phi_{\phi}$ the phase of the electric potential. Radial density transport (in the $J_{0}=1$ limit) is

$$
\Gamma=\left\langle\delta n \frac{\partial \phi}{\partial \alpha}\right\rangle_{\alpha}
$$

Therefore, for a given amplitude of perturbations, the flux is maximum for a phase-shift $\Phi_{n, W}-\Phi_{\phi}= \pm \pi / 2$, and zero for a zero phase-shift.

We hypothesize that for high-enough concentrations, the impurity plays enough role in 

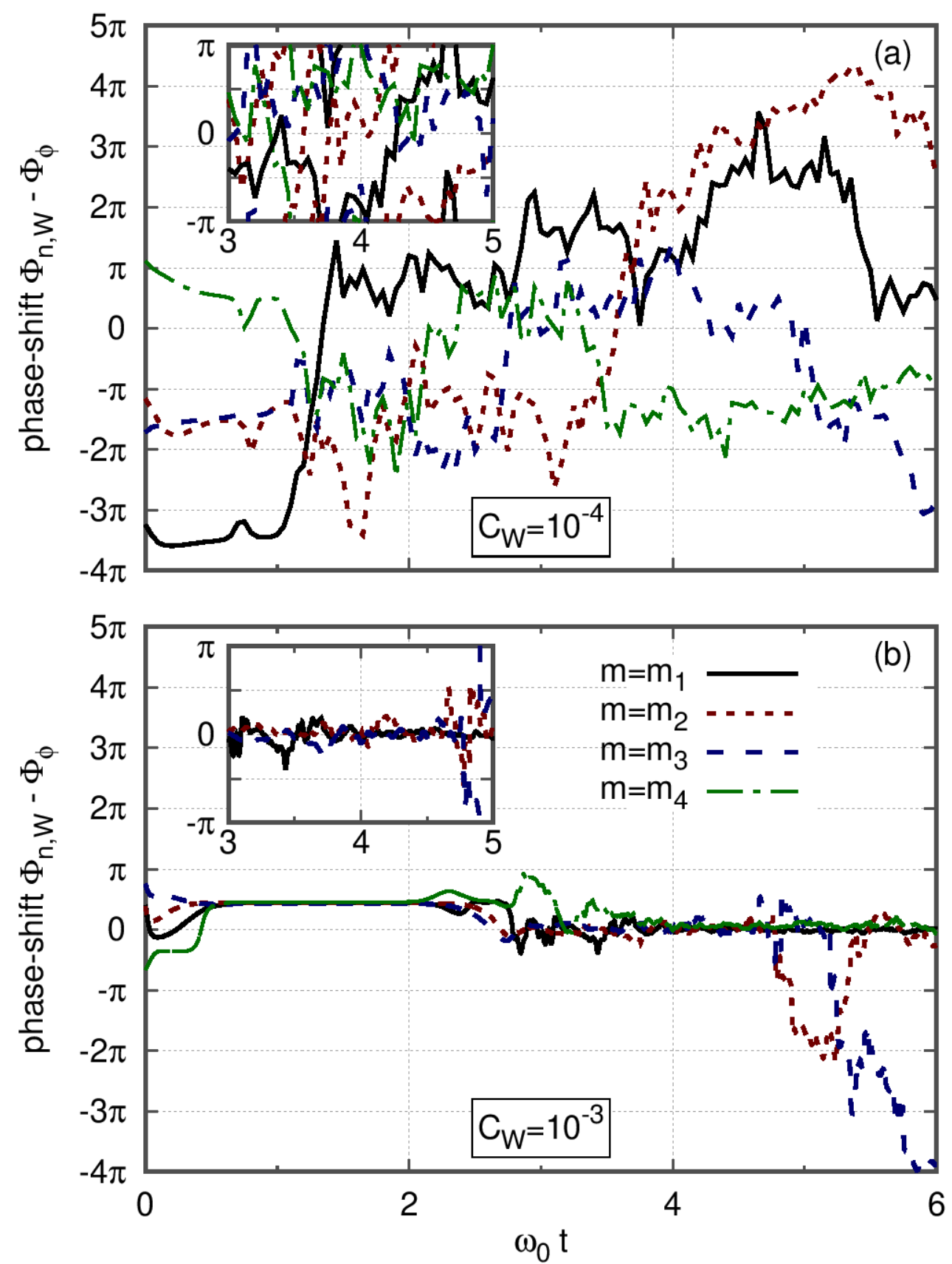

FIG. 11. Time-evolution of the difference between the phase of tungsten density fluctuations $\Phi_{n, W}$, and the phase of electric potential fluctuations $\Phi_{\phi}$. The legend is shared between the figures: the modes $m_{1}$ to $m_{4}$ correspond to the four modes with the highest average amplitude (where the mode $m_{1}$ is the strongest, $m_{2}$ the second strongest, etc.) in the time interval $3<\omega_{0} t<5$. (a) Tungsten concentration $C_{W}=10^{-4}$ (just below the transition). In this case, $m_{1}=4, m_{2}=7$, $m_{3}=6$, and $m_{4}=5$. (b) Tungsten concentration $C_{W}=10^{-3}$ (just above the transition). In this case, $m_{1}=10, m_{2}=12, m_{3}=13$, and $m_{4}=9$. Insets: zoom on the time interval $3<\omega_{0} t<5$ where the fluxes are measured (with an alternative representation based on modulo $2 \pi$ ). 
the turbulence that it can drive a phase-synchronization between potential fluctuations and its own density fluctuations. Let us focus on case 1 for example. Note that the value of the beginning of the transition, $C_{W}=2 \times 10^{-4}$, corresponds to $C_{W} Z_{W}^{2}=0.32$, at which point the contributions of tungsten and deuterium in the quasineutrality equation, Eq. (3), become comparable in amplitude.

To test this hypothesis, we focus on two values of concentration within case 1, just

below $\left(C_{W}=10^{-4}\right)$ and just above $\left(C_{W}=10^{-3}\right)$ the transition. Fig. 11 shows the phase differential between tungsten density fluctuations and electric potential fluctuations (against time). During the linear phase, which corresponds to $\omega_{0} t<1$ for $C_{W}=10^{-4}$ and $\omega_{0} t<2.3$ for $C_{W}=10^{-3}$, the phase differential stays more or less locked to $\pi / 2[2 \pi]$, for both values of concentration, although the locking appears to be stronger for $C_{W}=10^{-3}$. Later, in the nonlinear, steady-state, the qualitative difference between the two cases is much more pronounced. For low concentration, the phase-shift appears to be essentially random. In contrast, for high concentration, the phase-shift for the dominant modes is roughly locked to 0, which indicates a synchronization phenomenon between impurity density perturbations and potential perturbations, which effectively quenches transport. We found qualitatively similar results for other values of $C_{W}$.

In the case $C_{W}=10^{-3}$, we further analyzed the relationship between the phase-shifts and the amplitudes of the modes. It appears that temporary significant departures from a zero phase-shift, such as for $m=m_{3}=13$ at $\omega_{0} t>5$, correspond to temporary decreases in the mode amplitude. In other words, for a given mode, synchronization is robust as long as its amplitude is significant in the turbulent spectrum.

For concentrations below $10^{-4}$, the results are qualitatively similar to those for $C_{W}=$ $10^{-4}$, and for concentrations above $10^{-3}$, the results are qualitatively similar to those for $C_{W}=10^{-3}$. This synchronization, which occurs only for high enough impurity concentrations, appears to be at the origin of the abrupt transition between passive and active transport.

\section{CONCLUSION}

In this work, we applied gyrokinetic numerical simulations of trapped-particle dynamics to investigate the limit of validity of the passive treatment of tungsten impurities. To this 


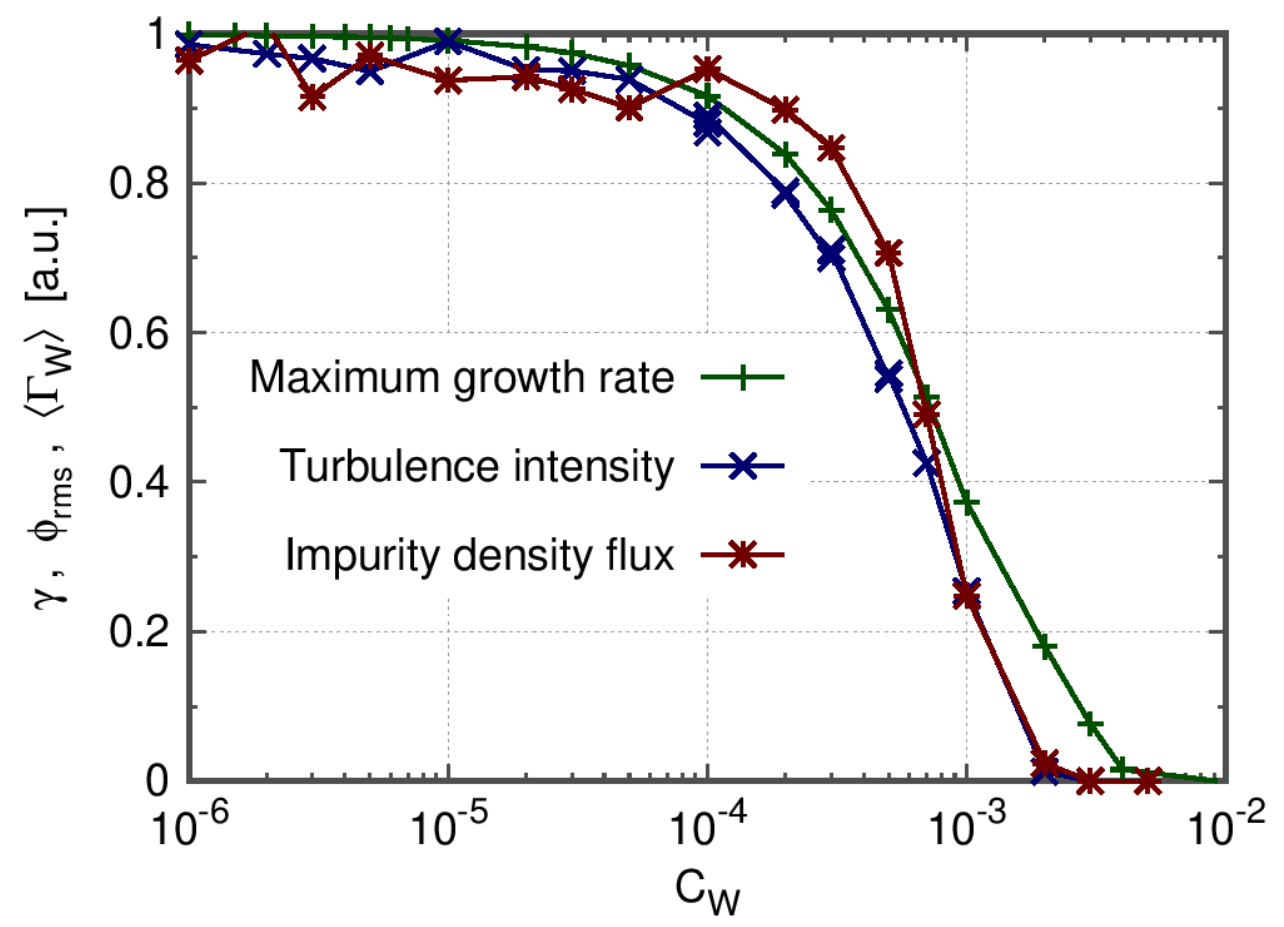

FIG. 12. Summary of results for case 1: linear growth-rate, turbulence intensity $\left(\phi_{\mathrm{rms}}\right)$, and density flux, all versus $\mathrm{W}^{40+}$ tungsten concentration. All 3 quantities are normalized by their limit as $C_{W} \rightarrow 0$.

aim, we compared simulations where the impurity is included in the quasineutrality equation (active treatment) with simulations where it is not (passive treatment). Since the passive treatment corresponds to the limit $C_{W} \rightarrow 0$, this work can also be taken as a simple scan of $C_{W} \cdot$

Fig. 12 summarizes the impact of tungsten concentration in the case where density gradient and temperature gradient are equal. It includes the linear growth rate, turbulence intensity, and impurity density flux, all normalized to their limit as $C_{W} \rightarrow 0$. There is a transition for all linear growth rate, turbulence intensity, and impurity density flux, centered around $C_{W} Z_{W}^{2} \approx 1.1$. However, the transition is significantly steeper for the flux. Around the middle of the transition (corresponding to the value 0.5 on the vertical axis in Fig. 12), the impact of tungsten concentration scales as $C_{W}^{0.80}$ for the linear growth rate, as $C_{W}^{0.82}$ for the turbulence intensity, and like $C_{W}^{1.35}$ for the impurity flux.

We investigated the physical mechanism responsible for this difference in behavior between turbulence intensity and transport. Our analysis indicates that phase-synchronization 


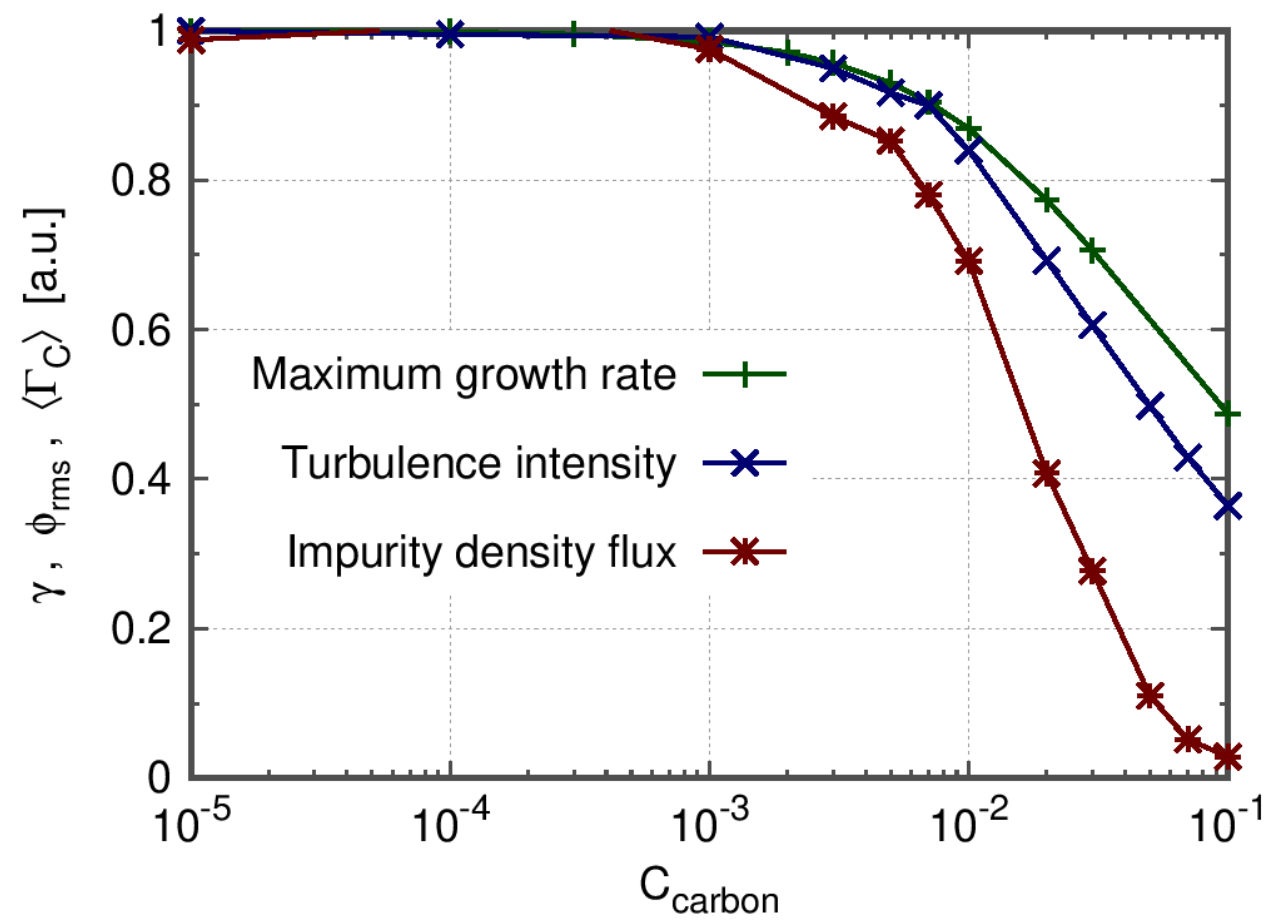

FIG. 13. Summary of results for $\mathrm{C}^{6+}$ carbon, with equal density and temperature gradients.

between impurity density fluctuations and electric potential fluctuations occurs for high enough impurity concentrations. This synchronization quenches impurity transport.

The steep transition for the turbulent flux may be viewed as a threshold, which gives the range of validity of the passive treatment, $C_{W}<2 \times 10^{-4}$. When the temperature gradient is finite and the density profile is flat, we obtain qualitatively similar results, except for the density flux which simply vanishes. For the heat flux, the range of validity of the passive treatment is slightly smaller than for the case with equal gradients, $C_{W}<10^{-4}$.

Finally, we have performed a similar analysis for $\mathrm{C}^{6+}$ carbon, although not as thoroughly as for tungsten. Fig. 13 summarizes the impact of carbon concentration in the case where density gradient and temperature gradient are equal. There are some qualitative differences compared with the tungsten case: 1 . the transition for the flux is centered around $C Z^{2} \approx 0.5$ (instead of around 1.1), and 2. the transition for the flux is at lower concentration than the transition for turbulence intensity and growthrate, which is opposite to the tungsten case. However, our main conclusion stands for carbon as well as for tungsten: around a concentration $C_{\text {impurity }} \sim 1 / Z^{2}$, there is a transition for all linear growth rate, turbulence intensity, and impurity density flux, and the transition is steeper for the flux than for 
turbulence, and steeper for turbulence than for the growth-rate.

Given that, for our parameters, the frequency of dominant modes can be comparable to the bounce frequency of tungsten, our results should be checked for larger system size, or with a non-bounce-averaged gyrokinetic model.

\section{ACKNOWLEDGMENTS}

This work was carried out within the framework of the French Federation for Magnetic Fusion Studies (FR-FCM) and of the EUROfusion Consortium and has received funding from the Euratom research and training programme 2014-2018 under grant agreement No 633053 for the project WP17-ENR-CEA-02. The views and opinions expressed herein do not necessarily reflect those of the European Commission. This work was granted access to the HPC resources of IDRIS under Allocation No 2017-27862 made by GENCI and of EXPLOR under project No 2017M4XXX0251. We are grateful for stimulating discussions with the participants in the Festival de Théorie.

\section{Appendix: Analytic estimation of the linear impact of impurity concentration}

The impact of impurities on the linear growth rate in the limit of low impurity concentrations can be estimated analytically. We write the dispersion relation formally as $D\left[\omega\left(C_{W}\right), C_{W}\right]=0$, where $\omega=\omega_{r}+\imath \gamma$ is the complex frequency. We define a complex coefficient $\beta$ as

$$
\beta=\left.\frac{\mathrm{d} \omega}{\mathrm{d} C_{W}}\right|_{C_{W} \rightarrow 0} .
$$

Then, $\beta$ is estimated from the following relationship,

$$
\left.\frac{\mathrm{d} D}{\mathrm{~d} C_{W}}\right|_{C_{W}=0}=\left.\frac{\partial D}{\partial C_{W}}\right|_{C_{W}=0}+\left.\beta \frac{\partial D}{\partial \omega}\right|_{C_{W}=0}=0 .
$$

We obtain $\beta=\beta_{N} / \beta_{D}$, with $\beta_{N}=\left.\frac{\partial D}{\partial C_{W}}\right|_{C_{W}=0}$ and $\beta_{D}=-\left.\frac{\partial D}{\partial \omega}\right|_{C_{W}=0}$

In Fourier space, the linearized Vlasov equation yields

$$
\hat{f}_{m, s}(\psi)=\frac{m \partial f_{\mathrm{eq}, s} / \partial \psi}{\omega_{r}-m \Omega_{d} E / Z_{s}+\imath \gamma} \hat{J}_{0, s} \hat{\phi}_{m}(\psi)
$$


where $\hat{J}_{0, s}$ is the Fourier equivalent of the operator $J_{0, s}$. In this linear analysis, we assume a strictly positive growth rate, $\gamma>0$. Substituting the latter linear response into the quasi-neutrality equation, Eq. (3), in Fourier space, yields

$$
\sum_{s} \frac{e Z_{s}^{2} n_{e q, s}}{T_{e q, s}}\left[\frac{1-f_{t}}{f_{t}}-\hat{\Delta}_{s}\right]=4 \pi \sqrt{2} \sum_{s} \frac{Z_{s}}{m_{s}^{3 / 2}} \int_{0}^{\infty} \frac{\hat{J}_{0, s}^{2} \partial f_{\mathrm{eq}, s} / \partial \psi}{\omega / m-\Omega_{d} E / Z_{s}} E^{1 / 2} \mathrm{~d} E
$$

for $m \neq 0$, where $\hat{\Delta}_{s}$ is the Fourier equivalent of the operator $\bar{\Delta}_{s}$. To the zeroth order in $\kappa_{n, T}\left(\psi / L_{\psi}\right)$ of $f_{e q, s}(\psi, E)$, we obtain the local dispersion relation [28],

$$
D=\sum_{s} Z_{s}^{2} C_{s} \tau_{s} D_{s}=0
$$

where

$$
\begin{gathered}
D_{s}=C_{n, s}-\kappa_{T} I_{r, s}-\left[C_{s} \kappa_{n}+\kappa_{T}\left(x_{s}-\frac{3}{2}\right)\right] I_{d, s}, \\
C_{n, s}=\frac{a}{R_{0}}\left[\frac{1-f_{t}}{f_{t}}+\left(\frac{m q_{0} \rho_{c, s}}{2 L_{\psi}}\right)^{2}+\left(\frac{k_{\psi} \delta_{b, s}}{2}\right)^{2}\right], \\
I_{r, s}=\frac{2}{\sqrt{\pi}} \int_{0}^{\infty} \hat{J}_{0, s}^{2}\left(\xi_{s}\right) e^{-\xi_{s}} \xi_{s}^{1 / 2} \mathrm{~d} \xi_{s}, \\
I_{d, s}=\frac{2}{\sqrt{\pi}} \int \frac{\xi_{s}^{1 / 2} e^{-\xi_{s}}}{\xi_{s}-x_{s}} \hat{J}_{0, s}^{2}\left(\xi_{s}\right) \mathrm{d} \xi_{s},
\end{gathered}
$$

$\xi_{s}=E / T_{0, s}$, and $x_{s}=Z_{s} \tau_{s} \omega /\left(m \Omega_{d}\right)$. In the limit of no impurities, we recover the dispersion relation of Refs. [33, 39].

Carrying out the integration in Eq. A.8) yields

$$
I_{r, s}=1-3(b+c)+\frac{15}{4}\left(b^{2}+c^{2}+4 b c\right)-\frac{105}{4} b c(b+c)+\frac{945}{16}(b c)^{2},
$$

where $b=\left[\left(k_{\psi} \delta_{b, s}\right) / 2\right]^{2}$ and $c=\left[\left(m q_{0} \rho_{c, s}\right) /\left(2 L_{\psi}\right)\right]^{2}$. Carrying out the integration in Eq. A.9 yields

$$
\begin{aligned}
\frac{I_{d, s}}{2}=\left(1-b x_{s}\right)^{2}\left(1-c x_{s}\right)^{2} & {\left[\frac{Z_{s}}{\left|Z_{s}\right|} \sqrt{-\pi x_{s}} e^{-x_{s}}-2 \sqrt{x_{s}} W\left(\sqrt{x_{s}}\right)\right] } \\
+\left(1-b P_{1}\right. & \left.+b^{2} P_{2}\right)^{2}\left(1-c P_{1}+c^{2} P_{2}\right)^{2} \\
& +2 b c\left[1-x_{s}+\frac{b+c}{2}\left(P_{2}-\frac{5}{4}\right)\right]+(b c)^{2} P_{3}
\end{aligned}
$$


where $W$ is the Dawson integral [45, 46],

$$
W(z)=\frac{1}{2} \sqrt{\pi} e^{-z^{2}} \operatorname{erfi}(z),
$$

and $P_{1}, P_{2}$ and $P_{3}$ are polynomials in $x_{s}$,

$$
\begin{aligned}
& P_{1}=1+2 x, \\
& P_{2}=\frac{3}{4}+\frac{x}{2}+x^{2}, \\
& P_{3}=6+\frac{9 x}{8}-x^{2}-\frac{x^{3}}{2} .
\end{aligned}
$$

In this paper, $C_{e}=1$ and $C_{D}=1-Z_{W} C_{W}$, therefore

$$
\beta_{N}=\left.Z_{W}^{2} \tau_{W} D_{W}\right|_{C_{W}=0}+Z_{W} \tau_{D}\left(\kappa_{n} I_{d, D}-\left.D_{D}\right|_{C_{D}=1}\right)
$$

Substituting the dispersion relation for $C_{W}=0, \tau_{e} D_{e}+\tau_{D} D_{D}=0$, we obtain an alternative expression for the numerator of $\beta$,

$$
\beta_{N}=\left.Z_{W}^{2} \tau_{W} D_{W}\right|_{C_{W}=0}+Z_{W} \tau_{D} \kappa_{n} I_{d, D}+Z_{W} \tau_{e} D_{e}
$$

The denominator $\beta_{D}$ satisfies

$$
\begin{aligned}
m \Omega_{d} \beta_{D}=\tau_{D}^{2} \kappa_{T, D} I_{d, D}- & \tau_{e}^{2} \kappa_{T, e} I_{d, e} \\
& +\tau_{D}^{2}\left[C_{D} \kappa_{n, D}+\kappa_{T, D}\left(x_{D}-\frac{3}{2}\right)\right] \frac{\mathrm{d} I_{d, D}}{\mathrm{~d} x_{D}} \\
& \quad-\tau_{e}^{2}\left[\kappa_{n, e}+\kappa_{T, e}\left(x_{e}-\frac{3}{2}\right)\right] \frac{\mathrm{d} I_{d, e}}{\mathrm{~d} x_{e}} .
\end{aligned}
$$

Given the relationships between the banana widths and Larmor radii of the species, and since $m \gg L_{\psi} k_{\psi} \sim \pi$, we choose to develop these expression to the first order in $\left(k_{\psi} \delta_{b, D}\right)^{2}$ and $\left(m \rho_{c, D} / L_{\psi}\right)^{2}$, but to the zeroth order in $\left(k_{\psi} \delta_{b, W}\right)^{2},\left(m \rho_{c, W} / L_{\psi}\right)^{2},\left(k_{\psi} \delta_{b, e}\right)^{2}$ and $\left(m \rho_{c, e} / L_{\psi}\right)^{2}$. Therefore, for $s=W$ and $s=e$ we approximate $D_{s}$ as

$$
D_{s}=\frac{a}{R_{0}} \alpha_{t}-\kappa_{T}-\left[C_{s} \kappa_{n}+\kappa_{T}\left(x_{s}-\frac{3}{2}\right)\right] I_{d, s},
$$

where $\alpha_{t}=\left(1-f_{t}\right) / f_{t}$. For $s=W$ and $s=e$ we approximate $I_{d, s}$ as

$$
I_{d, s}=2-2 Y_{s}\left(x_{s}\right)
$$

with

$$
Y_{s}\left(x_{s}\right)=e^{-x_{s}} \sqrt{\pi x_{s}}\left[\frac{Z_{s}}{\left|Z_{s}\right|} \imath-\operatorname{erfi}\left(\sqrt{x_{s}}\right)\right]
$$


and we approximate the derivative of $I_{d, s}$ as

$$
\frac{\mathrm{d} I_{d, s}}{\mathrm{~d} x_{s}}=\frac{\left(1 / 2-x_{s}\right) I_{d, s}-1}{x_{s}} .
$$

For deuterium, we approximate $I_{d, D}$ as

$$
I_{d, D}=2+2 Y_{D}\left(x_{D}\right)\left(1-2 d_{D} x_{D}\right)-2 d_{D}\left(1+2 x_{D}\right)
$$

where $d_{D}=\left[\left(k_{\psi} \delta_{b, D}\right) / 2\right]^{2}+\left[\left(m q_{0} \rho_{c, D}\right) /\left(2 L_{\psi}\right)\right]^{2}$, and we approximate the derivative of $I_{d, D}$ as

$$
\frac{\mathrm{d} I_{d, D}}{\mathrm{~d} x_{D}}=4 d_{D}(x-1)-2+2 Y_{D}\left(x_{D}\right)\left[\frac{1}{2 x_{D}}-1+d_{D}\left(2 x_{D}-3\right)\right] .
$$

This development finally yields

$$
\begin{aligned}
\frac{\beta}{4 m d_{D} x_{D} Z_{W}}=\kappa_{n} \frac{1+2 x_{D}\left[1+Y_{D}\left(x_{D}\right)\right]}{G} & -\frac{\eta}{2 d_{D} G} \\
& -x_{D} \eta \frac{Q_{2, p}\left(x_{D}\right)+Q_{2, D}\left(x_{D}\right) Y_{D}\left(x_{D}\right)}{G^{2}},
\end{aligned}
$$

where

$$
\begin{gathered}
G=8 x_{D}^{2} \kappa_{T}+Q_{2}\left(x_{D}\right) Y_{D}\left(x_{D}\right)+Q_{2}\left(x_{e}\right) Y_{e}\left(x_{e}\right), \\
\eta=\frac{a}{R_{0}}\left(1+Z_{W}\right) \alpha_{t}+2 \kappa_{n} Y_{D}\left(x_{D}\right) \\
+Q_{1}\left(x_{e}\right)-Q_{1, e}\left(x_{e}\right) Y_{e}\left(x_{e}\right) \\
\quad+Z_{W}\left[Q_{1}\left(x_{W}\right)-Q_{1, W}\left(x_{W}\right) Y_{W}\left(x_{W}\right)\right],
\end{gathered}
$$

and $Q_{i}$ are polynomials,

$$
\begin{gathered}
Q_{1}(x)=2 \kappa_{T} x+2 \kappa_{T}, \\
Q_{1, e}(x)=2 \kappa_{T} x+\left(2 \kappa_{n}-3 \kappa_{T}\right), \\
Q_{1, W}(x)=2 \kappa_{T} x-3 \kappa_{T}, \\
Q_{2}(x)=4 \kappa_{T} x^{2}+\left(4 \kappa_{n}-12 \kappa_{T}\right) x+\left(3 \kappa_{T}-2 \kappa_{n}\right), \\
Q_{2, p}(x)=4 \kappa_{T} x^{2}+\left(4 \kappa_{n}-14 \kappa_{T}\right) x+\left(4 \kappa_{T}-4 \kappa_{n}\right), \\
Q_{2, D}(x)=4 \kappa_{T} x^{2}+\left(4 \kappa_{n}-16 \kappa_{T}\right) x+\left(9 \kappa_{T}-6 \kappa_{n}\right) .
\end{gathered}
$$

Substituting the input parameters yields $\beta=-1206-22273$ for case 1 , and $\beta=155-$ 8938 for case 2 . These results can be compared with the values obtained numerically from 
the full dispersion relation. We find that the relative inaccuracy in terms of $|\beta|$ is $5.0 \%$ for case 1 and $11.6 \%$ for case 2 .

[1] T. Pütterich et al., Nucl. Fusion 50, 025012 (2010).

[2] C. Angioni et al., Nucl. Fusion 54, 083028 (2014).

[3] F. Casson et al., Plasma Phys. Control. Fusion 57, 014031 (2014).

[4] A. Loarte et al., Phys. Plasmas 22, 056117 (2015).

[5] C. Angioni et al., Nucl. Fusion 57, 056015 (2017).

[6] M. Greenwald et al., Phys. Plasmas 6, 1943 (1999).

[7] T. E. Evans et al., Nat. Phys. 2, 419 (2006).

[8] F. Köchl et al., Plasma Phys. Control. Fusion 60, 074008 (2018).

[9] A. M. Dimits et al., Phys. Plasmas 7, 969 (2000).

[10] X. Garbet, Y. Idomura, L. Villard, and T. Watanabe, Nucl. Fusion 50, 043002 (2010).

[11] S. Leerink et al., Phys. Rev. Lett. 109, 165001 (2012).

[12] T. Görler et al., Phys. Plasmas 21, 122307 (2014).

[13] G. Dif-Pradalier et al., Phys. Rev. Lett. 114, 085004 (2015).

[14] D. Estève et al., Nucl. Fusion 58, 036013 (2018).

[15] P. Donnel et al., Comput. Phys. Commun. 234, 1 (2019).

[16] P. Donnel et al., Plasma Phys. Control. Fusion 61, 044006 (2019).

[17] C. Angioni and A. Peeters, Phys. Rev. Lett. 96, 095003 (2006).

[18] S. Futatani, S. Benkadda, Y. Nakamura, and K. Kondo, Phys. Rev. Lett. 100, 025005 (2008).

[19] A. Skyman, H. Nordman, and P. Strand, Nucl. Fusion 52, 114015 (2012).

[20] H. Nordman et al., Plasma Phys. Control. Fusion 53, 105005 (2011).

[21] C. Angioni et al., Nucl. Fusion 57, 022009 (2016).

[22] B. Coppi, H. Furth, M. Rosenbluth, and R. Sagdeev, Phys. Rev. Lett. 17, 377 (1966).

[23] T. Fülöp and H. Nordman, Phys. Plasmas 16, 032306 (2009).

[24] S. Futatani et al., Phys. Rev. Lett. 109, 185005 (2012).

[25] W. Guo, L. Wang, and G. Zhuang, Phys. Plasmas 23, 112301 (2016).

[26] H. Du, Z.-X. Wang, J. Q. Dong, and S. F. Liu, Phys. Plasmas 21, 052101 (2014).

[27] H. Du, Z.-X. Wang, and J. Q. Dong, Phys. Plasmas 23, 072106 (2016). 
[28] M. Idouakass et al., Phys. Plasmas 25, 062307 (2018).

[29] N. Bonanomi et al., Nucl. Fusion 58, 036009 (2018).

[30] R. Neu et al., Phys. Plasmas 20, 056111 (2013).

[31] M. Tagger, G. Laval, and R. Pellat, Nucl. Fusion 17, 109 (1977).

[32] H. Biglari, P. H. Diamond, and P. W. Terry, Phys. Fluids 31, 2644 (1988).

[33] G. Depret, X. Garbet, P. Bertrand, and A. Ghizzo, Plasma Phys. Control. Fusion 42, 949 (2000).

[34] Y. Sarazin et al., Plasma Phys. Control. Fusion 47, 1817 (2005).

[35] G. Darmet et al., Commun. Nonlinear Sc. Numerical Simulation 13, 53 (2008), vlasovia 2006: The Second International Workshop on the Theory and Applications of the Vlasov Equation.

[36] E. Gravier et al., Phys. Plasmas 26, 082306 (2019).

[37] T. Cartier-Michaud, P. Ghendrih, V. Grandgirard, and G. Latu, ESAIM: Proc. 43, 274 (2013).

[38] T. Cartier-Michaud et al., J. Phys.: Conf. Series 561, 012003 (2014).

[39] T. Drouot et al., Euro. Phys. J. D 68, 280 (2014).

[40] T. Drouot et al., Phys. Plasmas 22, 082302 (2015).

[41] M. Lesur et al., Phys. Plasmas 24, 012511 (2017).

[42] R. Dominguez and M. Rosenbluth, Nucl. Fusion 29, 844 (1989).

[43] R. Paccagnella, F. Romanelli, and S. Briguglio, Nucl. Fusion 30, 545 (1990).

[44] J. Dominski et al., in Proc. IAEA Fusion Energy Conf., 2018 (IAEA, Vienna, 2018), pp. IAEA-CN-258/711.

[45] H. G. Dawson, Proceedings of the London Mathematical Society s1-29, 519 (1897).

[46] M. Abramowitz and I. A. Stegun, Applied Mathematics Series 55, 62 (1966). 